\title{
NEW MULTI-SCALE MODELS ON MESOSCALES AND SQUALL LINES*
}

\author{
ANDREW J. MAJDA ${ }^{\dagger}$ AND YULONG XING $\ddagger$
}

Dedicated to the sixtieth birthday of Professor Andrew Majda

\begin{abstract}
Squall lines are coherent turbulent traveling waves on scales of order $100 \mathrm{~km}$ in the atmosphere that emerge in a few hours from the interaction of strong vertical shear and moist deep convection on scales of order $10 \mathrm{~km}$. They are canonical coherent structures in the tropics and middle latitudes reflecting upscale conversion of energy from moist buoyant sources to horizontal kinetic energy on larger scales. Here squall lines are introduced through high resolution numerical simulations which reveal a new self-similarity with respect to the shear amplitude. A new multi-scale model on mesoscales which allows for large vertical shears, appropriate for squall lines, is developed here through systematic multi-scale asymptotics. Mathematical and numerical formulations of the new multi-scale equations are utilized to illustrate both new mathematical and physical phenomena captured by these new models. In particular, non-hydrostatic Taylor-Goldstein equations govern the upscale transports of momentum and temperature from the order $10 \mathrm{~km}$ microscales to the order $100 \mathrm{~km}$ mesoscales; surprisingly, upright single mode convective heating without tilts can lead to significant upscale convective momentum transport from the microscales to the mesoscales due to the strong shear. The multi-scale models developed here should be especially useful for dynamic parameterizations of upscale transports as well as for new theory in three-dimensions with a transverse shear component, where contemporary theoretical understanding is meager.
\end{abstract}

Key words. Squall lines, multi-scale asymptotics, Taylor-Goldstein equations, upscale convective momentum transport.

AMS subject classifications. $65 \mathrm{~T} 50,76 \mathrm{~F} 55,93 \mathrm{~A} 30$.

\section{Introduction}

Geophysical flows are a rich source of fascinating problems for applied mathematicians involving complex multi-scale nonlinear systems, where energy cascades upward from the small scales to the large scales through anisotropic processes involving vortices and gravity waves. On the other hand, the improved parameterization of unresolved features of moist tropical convection is a central challenge in current computer models for long range ensemble forecasting of weather and short term climate with large worldwide societal impact [41]. The reason for this is the observed multi-scale features of organized coherent tropical convection across a wide range of scales varying from tens of kilometers and a few hours to the planetary scale of order $40,000 \mathrm{~km}$ on intraseasonal time scales with significant energy transfer across these scales [20, 38, 42, 46]. Recent processing of observational data [38] suggests the statistical self-similarity of tropical convection from the smallest, shortest scales to organized mesoscale convective systems [19] to convective clusters to equatorial synoptic-scale superclusters to planetary/intraseasonal oscillations. For this reason, it is interesting to develop systematic multiscale asymptotic models [24, 25, 26, 30, 34] for the nonlinear cascade across scales in the tropics, and the first author has done this recently for the self-similar behavior from the microscales to mesoscales to planetary/intraseasonal scales $[31,32]$. Such quantitative models are useful for quantifying

\footnotetext{
*Received: July 13, 2008; accepted (in revised version): December 9, 2008.

$\dagger$ Department of Mathematics and Center for Atmosphere-Ocean Science, Courant Institute of Mathematical Sciences, New York University, New York, NY (jonjon@cims.nyu.edu).

$\ddagger$ Corresponding author. Department of Mathematics and Center for Atmosphere-Ocean Science, Courant Institute of Mathematical Sciences, New York University, New York, NY (xing@cims. nyu.edu).
} 
the observed multiscale behavior in, for example, tropical intraseasonal oscillations $[2,3,4,5,33,37]$.

The important spatio-temporal scales for cloud resolving modeling are the fully nonlinear scales with active moisture, involving spatio-temporal scales

$$
L_{m}=10 \mathrm{~km}, \quad T_{m}=15 \mathrm{~min} .
$$

The organized impact of moist convection on the scales in (1.1) through squall lines, tropical cyclogenesis, and other mesoscale convective systems [8, 19, 39, 43] occurs on the mesoscale spatio-temporal scales, $L_{M}$ and $T_{M}$, with

$$
L_{M}=100 \mathrm{~km}=\epsilon^{-1} L_{m}, \quad T_{M}=2.5 \mathrm{hrs}=\epsilon^{-1} T_{m}
$$

for $\epsilon \approx 0.1$. The spatial scale $L_{m}=10 \mathrm{~km}$ in $(1.1)$ is called the microscale below, while the scale $L_{M}=100 \mathrm{~km}$ in (1.2) is called the mesoscale. Contemporary numerical weather prediction (NWP) models [40] currently can have a finest mesh size on the order of $10 \mathrm{~km}=L_{m}$, so new numerical strategies that lead to the improved representation of the mesoscale impacts of tropical convection have central importance.

The topic of the present paper is the development of new multi-scale models on the spatio-temporal scales in (1.1) and (1.2), which provide potentially new insight into the multi-scale development of organized squall lines on mesoscales. Squall lines are coherent structures consisting of turbulent traveling waves which emerge on the mesoscales in (1.2) from the interactions of shear and moist convection on the smaller scales in (1.1). There is a beautiful far field theory for the upscale cascade effects of squall lines in [39], and numerous well-designed high resolution numerical simulations $[10,27,43,47,49]$ which illustrate various multi-scale aspects of squall line dynamics involving the two scales in (1.1) and (1.2). An elegant theory of moist gravity currents which gives insight into the propagation of long-lived squall lines in middle latitudes has been developed in [45]. The recent survey article for meteorologists [19] is strongly recommended to the interested reader. Despite all of this effort, the detailed multiscale mechanisms in squall lines are not fully understood.

The earlier work for studying squall lines in multi-scale models on mesoscales $[26,31]$ or the hurricane embryo $[35,36]$ involved two assumptions: low Froude number velocities and a weak temperature gradient (WTG). Here we develop new multiscale models which do not require low Froude numbers and are more realistic for the strong shears that occur in squall lines. The new multi-scale models both have new mathematical features and capture important additional physical phenomena compared with the earlier models. Here is the outline for the remainder of the paper. In section 2, we provide some new high resolution numerical simulations of squall lines for tropical jet shears which both serve as an introduction to squall line dynamics as turbulent multi-scale traveling waves for mathematicians and also demonstrate, for the first time, surprising self-similarity in squall line dynamics as the ambient shear strength changes. The new multi-scale models for squall lines are developed in section 3. Mathematical and numerical formulations of the resulting multi-scale equations are developed in section 4 together with simple applications illustrating the physical effects and new phenomena in these models. The paper ends with a brief concluding discussion.

\section{Tropical squall-lines - turbulent traveling waves}

In this section, we show some basic effects of typical tropical squall lines by three squall line simulations. We first present the moist anelastic equations which 
are utilized as the basic equations to generate a squall line. Then the background environment is carefully selected for mimicing the formation of a squall line in a few hours. Based on this background, three squall line simulations are run with different shears. Large scale effects and turbulent eddy fluxes are shown to demonstrate the main features of the propagating squall line.

2.1. Preliminaries: the moist anelastic equations. Here the moist nonhydrostatic anelastic equations with bulk cloud microphysics [17, 29] are utilized as the basic equations for the microscale dynamics. With suitable nondimensional units explained below [26], which have the space-time scales

$$
L_{m}=10 \mathrm{~km} \quad \text { and } \quad T_{m}=15 \mathrm{~min},
$$

with

$$
\left[\mathbf{u}_{h}\right]=[w]=10 \mathrm{~m} / \mathrm{s},
$$

these equations are given by the dynamical core

$$
\begin{aligned}
\frac{D \mathbf{u}_{h}}{D t} & =-\nabla_{h} p \\
\frac{D w}{D t} & =-p_{z}+\epsilon^{-1} \theta+\left(\bar{\epsilon} q_{v}-q_{r}-q_{c}\right) \\
\frac{D \theta}{D t}+N^{2}(z) \epsilon^{-1} w & =\epsilon^{-1} L \frac{\theta_{0}}{p_{0}}\left(C_{d}-E_{r}\right) \\
\operatorname{div}_{h} \mathbf{u}_{h}+\rho^{-1}(\rho w)_{z} & =0
\end{aligned}
$$

with

$$
\frac{D}{D t}=\frac{\partial}{\partial t}+\mathbf{u}_{h} \cdot \nabla_{h}+w \frac{\partial}{\partial z}
$$

and the cloud dynamic equations

$$
\begin{aligned}
\frac{D q_{v}}{D t} & =-C_{d}+E_{r} \\
\frac{D q_{c}}{D t} & =C_{d}-A_{r} \\
\frac{D q_{r}}{D t}-\frac{1}{\rho} \frac{\partial}{\partial z}\left(\rho V_{t} q_{r}\right) & =A_{r}-E_{r} .
\end{aligned}
$$

In $(2.2), \rho(z), N^{2}(z), \theta_{0}(z)$, and $p_{0}(z)$ are the nondimensional versions of the dry statically stable vertical profile $[26,34]$. The potential temperature in $(2.2)$ is scaled as small deviation from hydrostatic balance; that is, the units of $\theta$ are $3 \mathrm{~K}$, which compares with a dry lapse rate of $30 \mathrm{~K}$ over $10 \mathrm{~km}$; thus $\epsilon \approx 0.1$ in $(2.2)$ (see the appendix for more detailed discussion and $[26,31,35]$ for additional information). The quantities $q_{v}, q_{c}$, and $q_{r}$ are the mixing ratios for cloud vapor, water, and rain, respectively, rescaled by the factor $\epsilon^{-2}$. This rescaling developed in [26] renders the potential temperature equation in (2.2) dimensionless with order-1 latent heat prefactor $\mathrm{L}$ and simultaneously guarantees that the condensation of cloud vapor, $C_{d}$, evaporation of rain, $E_{r}$, and the conversion of cloud water to rain by both autoconversion and collection, $A_{r}$, as well as the fall velocity, $V_{T}$, are order one processes on the time scales in (2.1). While the detailed forms of $C_{d}, E_{r}$, and $A_{r}[8,17]$ are not 
needed for the discussion here, the tacit standard assumption utilized in achieving a single time scale governing the moisture source terms in (2.2) and (2.4) is that the very fast processes associated with supersaturated water vapor are equilibrated by constraining the cloud vapor to always lie below or equal to saturation [17, 26, 29]. For simplicity in exposition, all other source terms and dissipation in (2.2) and (2.4) have been set to zero; they are readily added in the analysis below. The equations in (2.2) and (2.4) have been utilized extensively as the microscale dynamical equations in both cloud-resolving modeling and superparameterization [11, 12, 13, 15], so they are a natural starting point for this paper.

2.2. The squall line environment. In the remaining part of this paper, as discussed above, non-dimensional units are used with the following reference scales, except for some cases where detailed explanations are provided.

\begin{tabular}{lll} 
Parameter & Nondimensional units & Description \\
\hline $\mathrm{L}$ & $10 \mathrm{~km}$ & length scale \\
$\mathrm{t}$ & $15 \mathrm{~min}$ & time scale \\
$\mathrm{u}, \mathrm{w}$ & $10 \mathrm{~km} / 15 \mathrm{~min} \approx 10 \mathrm{~m} / \mathrm{s}$ & horizontal \& vertical velocity scale \\
$\theta$ & $3 \mathrm{~K}$ & potential temperature scale \\
\hline
\end{tabular}

The squall line experiment designed in [14] is explored here. The model we solve is a $2 \mathrm{D}$ version of $(2.2)$, where everything is a function of $\mathrm{x}, \mathrm{z}$ only, and the horizontal velocity $\mathbf{u}_{h}$ is reduced to the east/west component $u_{h}$. The experiment has a $2 \mathrm{D}$ domain of $1024 \mathrm{~km}$ length and $25 \mathrm{~km}$ height. The grid employed here is a uniform 2 -km horizontal grid and 0.25 -km vertical grid, with a gravity wave absorber applied in the uppermost $7 \mathrm{~km}$ of the domain. An open lateral boundary condition is used. The initial temperature, humidity profiles, and horizontal wind fields are based on the GARP GATE Phase-III mean sounding. A 4-km-deep, 512-km-long cold pool of $D \theta^{\prime}=-6.75 \mathrm{~K}$ and $D q_{v}^{\prime}=-3.5 \mathrm{~g} \mathrm{~kg}^{-1}$ is placed in the domain on the initial data to initiate convection. This initial cold pool, which was prescribed as a negative potential temperature and moisture perturbation on the initial condition as shown in figure 2.1, is set to mimic the background cold air produced by a decaying cold front in the synoptic environment [21].

A large-scale forcing representing climatological background, as shown in figure 2.2 , is imposed on the model through the cooling and moistening rates. We keep this large-scale forcing for 6 hours, then remove it and observe whether a squall line forms and keeps propagating for many hours afterwards as a turbulent free wave. To provide small-scale excitation (important for the initial development of convection), a $10 \%$ amplitude random noise is added to the surface fluxes, with random numbers generated at every model time step.

Three different initial large scale background shears, with same shape and different magnitude, are used to generate the squall lines. They are given by

$$
\bar{U}(z)= \begin{cases}a\left(\cos \left(\frac{\pi z}{1.2}\right)-\cos \left(\frac{2 \pi z}{1.2}\right)\right) & \text { if } z<1.2, \\ -2 a & \text { otherwise }\end{cases}
$$

for a fixed coefficient $a$. We show the case when $\mathrm{a}=1$ in figure 2.3. The steering level refers to the height where the phase speed is equal to the squall line flow speed of the emerging wave. It is marked in figure 2.3, based on the squall line speed computed from the experiment below. Next, we run this squall line experiment in the same thermodynamic environment for three different cases with $\mathrm{a}=1,0.8$ and 0.5 , 

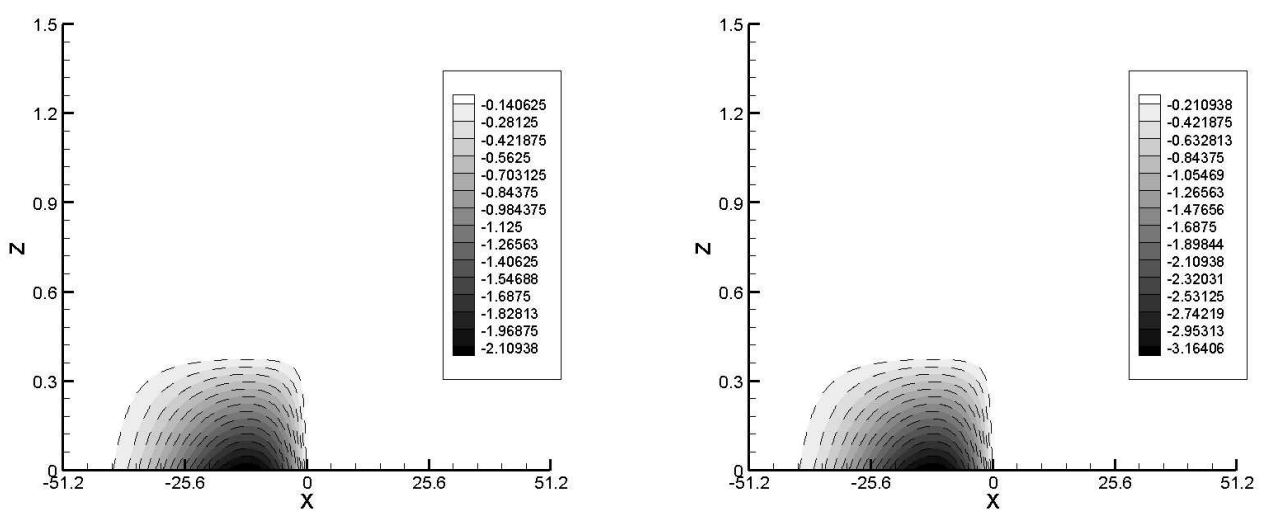

FIG. 2.1. The contours of the initial cold pool. Left: Potential temperature; Right: Specific humidity.

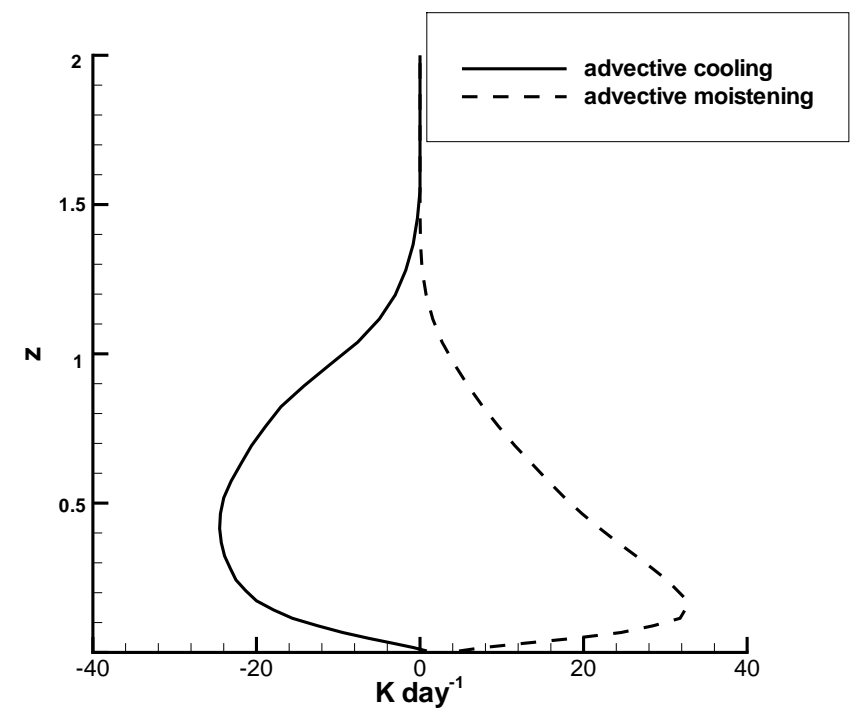

FIG. 2.2. Vertical profiles of the prescribed large scale advective cooling and moistening rates used in the experiments.

and compare these results where we systematically reduce the ambient shear and thus raise the Richardson number ([30], chapter 3$)$. It is interesting that a propagating squall line always emerges in these three cases with speed determined by the exact same steering level. We have repeated the experiments with no initial cold pool imposed, and the same steering level is observed. Further experiments demonstrate that the steering level changes as we modify either cooling or moistening profiles. Therefore, the steering level is independent of the magnitude of background shears and the initial cold pool condition, but depends on the background thermodynamics. If the squall line is non-traveling, it is easier for us to analyze the data. For this 


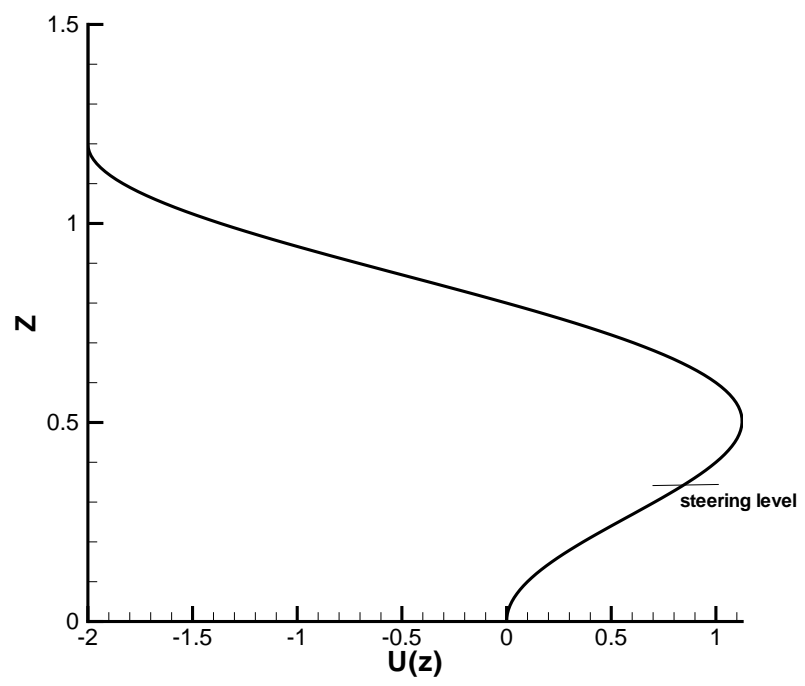

FIG. 2.3. Plot of $\bar{U}(z)$. The steering level for the squall lines is at roughly $3.5 \mathrm{~km}$ in height.

purpose, we subtract the speed of the steering level from the initial large scale shear in our experiment, and this is the background shear we actually use and get steady turbulent fronts.

2.3. The squall line simulations. With the above background information, we report in detail on the three squall line simulations. The stopping time is set as 36 hours, when the squall line remains statistically steady for a long time. This can be clearly observed from the space-time contours of the surface precipitation shown in the top left of figure 2.4, 2.5 and 2.6. In table 2.1, we show the velocity of the steering level (also noted as squall line speed) and the maximum of the original background shear before we subtract the squall line speed, defined as jet max here, from which we observe that the steering level keeps the same for all these experiments. This shows that if we only change the magnitude of the background shear and keep the same thermodynamic base state, the steering level will not change. However, we expect that if the shear becomes sufficiently weak, i.e., if a in (2.5) is small enough, the turbulent traveling wave will decay and will not propagate in time. We find that when $a=0.3$ or smaller, the wave dies after the initial formation. The corresponding surface precipitation is shown in figure 2.7 for $a=0.3$, from which we observe that for the first few hours the initial cold pool generates convection. It stays for few hours, after that it starts to die and disappears completely after the 20th hour. Note that the same background thermodynamic sounding is used in this case. This shows that even in a thermodynamic background favorable for the formation of a squall line, a weak enough background shear flow does not generate a squall line.

Next, we concentrate on large scale features in these simulations. As we can see from the surface precipitation contour plots, the three squall lines are statistically quasi steady as turbulent traveling waves after the initial phase. Hence we compute the time average over the five hours between the 18th and 23rd hours in the frame moving with the steering level wind, to gather time averaged statistical data. This 


\begin{tabular}{|c|c|c|c|}
\hline Experiment & $\mathrm{a}=1$ & $\mathrm{a}=0.8$ & $\mathrm{a}=0.5$ \\
\hline Jet max & 1.125 & $0.9=1.125 \times a$ & $0.5625=1.125 \times a$ \\
\hline Squall line speed & 0.825 & $0.66=0.825 \times a$ & $0.4125=0.925 \times a$ \\
\hline Steering level (height) & 0.34 & 0.34 & 0.34 \\
\hline
\end{tabular}

TABLE 2.1. The jet max, squall line speed, and steering level of three 2D CRM simulations.

time averaged numerical solution of horizontal velocity is denoted by $\langle u\rangle(x)$. Moreover the large scale velocity $\langle\bar{u}\rangle$, on mesoscales of order $100 \mathrm{~km}$ or 10 in nondimensional units, is defined as the spatial average

$$
\langle\bar{u}\rangle(x)=\frac{1}{9.6} \int_{-4.8}^{4.8}\langle u\rangle(x+s) d s,
$$

and the spatial fluctuation of velocity is given by

$$
\left\langle u^{\prime}\right\rangle(x)=\langle u\rangle(x)-\langle\bar{u}\rangle(x) .
$$

Since 3.2 is often chosen as the large scale resolution in the superparameterization test $[14,48], 9.6$ which corresponds to the length of 3 large scale cells, is used instead of 10 in (2.6). Similarly, we can compute $\langle\bar{\theta}\rangle,\left\langle\theta^{\prime}\right\rangle$ and the source terms $\left\langle\bar{S}_{\theta}\right\rangle,\left\langle S_{\theta}^{\prime}\right\rangle$ for the temperature equation in (2.2). This large scale data is shown in figure 2.4, 2.5 and 2.6 for different experiments. The plot of the source term $\left\langle\bar{S}_{w}\right\rangle$ for the vertical velocity is not included here, because it is one order smaller when compared with the source term $\left\langle\bar{S}_{\theta}\right\rangle$. Hence we concentrate on the source term $\left\langle\bar{S}_{\theta}\right\rangle$ in the following discussion, as it dominates the source terms. From these figures, we observe that the large scale horizontal velocities have very similar structures, due to the fact that the initial background shears share the same structure. The horizontal velocity has a shock-like structure with strong negative velocities at low levels in front but at high levels behind the squall line, i.e. a jump updraft [19]. To further explore the self-similarity, we compute the correlation between these plots after a phase shift aligning the center of the squall lines. The results are shown below in table 2.2. The correlation is very high, which shows very good structural agreement among them. We remind the reader that these large scale variables are the most important thing to examine in a squall line. Their structures are not affected by altering the strength of the background shears only and are qualitatively self-similar.

\begin{tabular}{|c|c|c|}
\hline & Between experiments a=1 and 0.8 & Between experiments $\mathrm{a}=1$ and 0.5 \\
\hline$\langle\bar{u}\rangle$ & 0.9278 & 0.8923 \\
\hline$\langle\bar{\theta}\rangle$ & 0.9002 & 0.8579 \\
\hline$\left\langle S_{\theta}\right\rangle$ & 0.8972 & 0.8431 \\
\hline
\end{tabular}

TABLE 2.2. The correlation between the large scale variables from these simulations.

Next, other useful statistical features of the squall line dynamics are presented. If we split the model into mesoscale and microscale, the upscale turbulent eddy fluxes $\overline{\left(\left\langle w^{\prime}\right\rangle\left\langle u^{\prime}\right\rangle\right)_{z}}$ and $\overline{\left(\left\langle w^{\prime}\right\rangle\left\langle\theta^{\prime}\right\rangle\right)_{z}}$ play an important role in the mesoscale, which will be shown later in section 3. Also, the fluctuation of the source terms $S_{\theta}^{\prime}$ and $S_{w}^{\prime}$ are important in determining the small scale structure. We then show the data: $\overline{\left(\left\langle w^{\prime}\right\rangle\left\langle u^{\prime}\right\rangle\right)_{z}}$, 

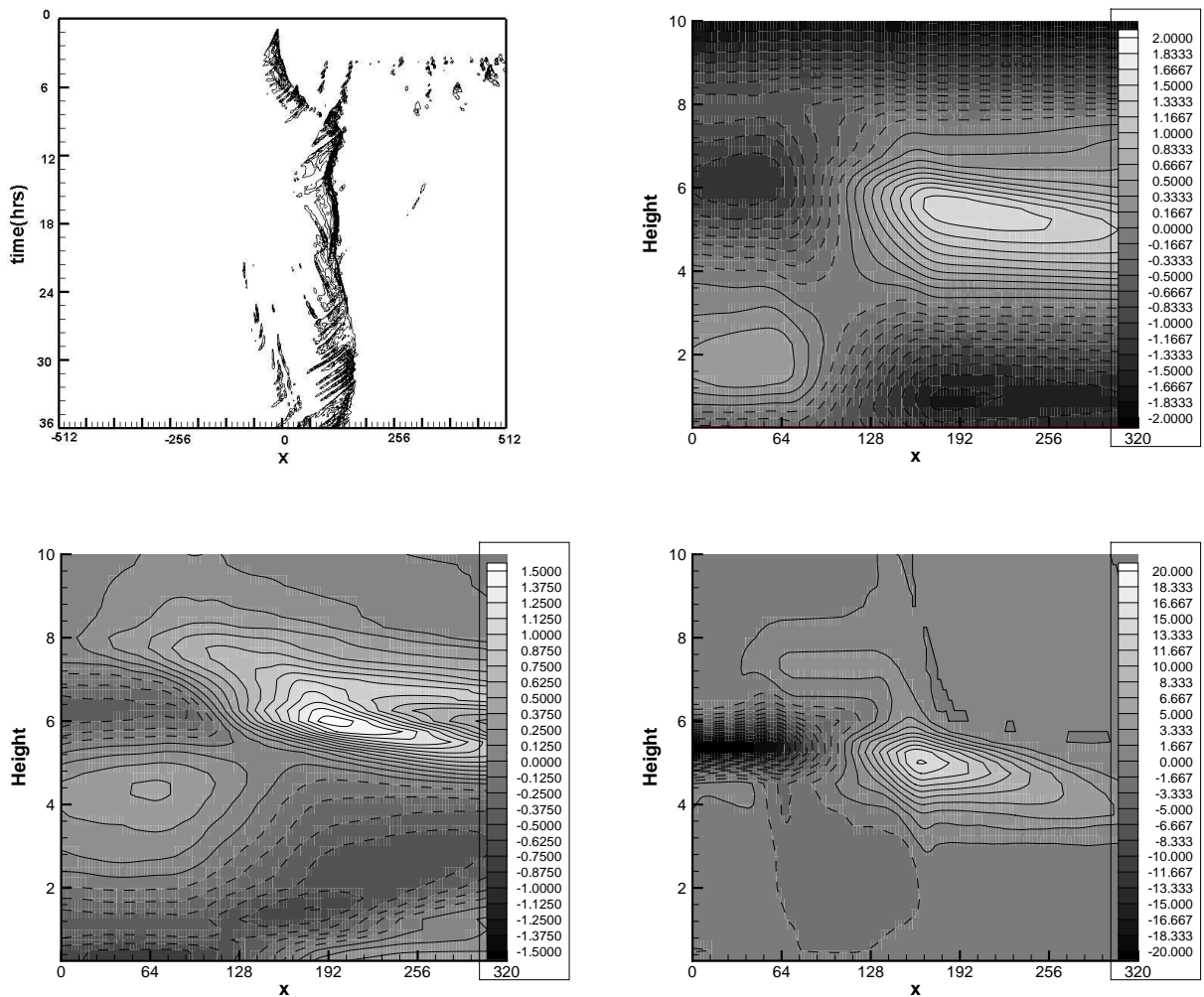

FIG. 2.4. The contours of the variables from the squall-line simulation with $a=1$. Top left: Surface precipitation; Top right: Large scale horizontal velocity $\langle\bar{u}\rangle$, averaged over five hours and $96 \mathrm{~km}$ domain as defined in (2.6); Bottom left: Large scale potential temperature $\langle\bar{\theta}\rangle ;$ Bottom right: Large scale source term $\left\langle\bar{S}_{\theta}\right\rangle$. Note: 1, the units for the $x$-axis and height are both $\mathrm{km}$ in these plots, rather that the non-dimensional units; 2, solid contour line represents positive value and dashed one represents negative value.

$\overline{\left(\left\langle w^{\prime}\right\rangle\left\langle\theta^{\prime}\right\rangle\right)_{z}},\left\langle S_{\theta}^{\prime}\right\rangle$ and $\left\langle S_{w}^{\prime}\right\rangle$, from these three experiments in figure 2.8, 2.9 and 2.10. We point out that for the upscale eddy flux $\overline{\left(\left\langle w^{\prime}\right\rangle\left\langle u^{\prime}\right\rangle\right)_{z}}$, very similar structures are observed by eye for the three simulations. This shows that within the same background thermodynamic sounding, if ambient shear strength changes the squall line experiments generate almost the same structure of upscale eddy flux $\overline{\left(\left\langle w^{\prime}\right\rangle\left\langle u^{\prime}\right\rangle\right)_{z}}$, but the magnitude diminishes with the shear strength parameter $a$.

A central issue for squall line dynamics is the following: depending on the nature of the thermodynamic background and the ambient shear, how much of the propagating squall line dynamics depends on the mean heating $\bar{S}_{\theta}$ compared with upscale turbulent cascades from the smaller scales reflected by the turbulent eddy fluxes described above. There is different role for these processes for different types of shears, as illustrated by several insightful numerical studies [9, 10, 27, 43, 47, 49], but this issue is not completely understood even in two space dimensions. For squall lines with three-dimensional transverse shear components, as often occur in nature, such theoretical understanding is meager at the present time [19]. This issue is important for parameterization and the main goal for the new models developed in section 3 is 

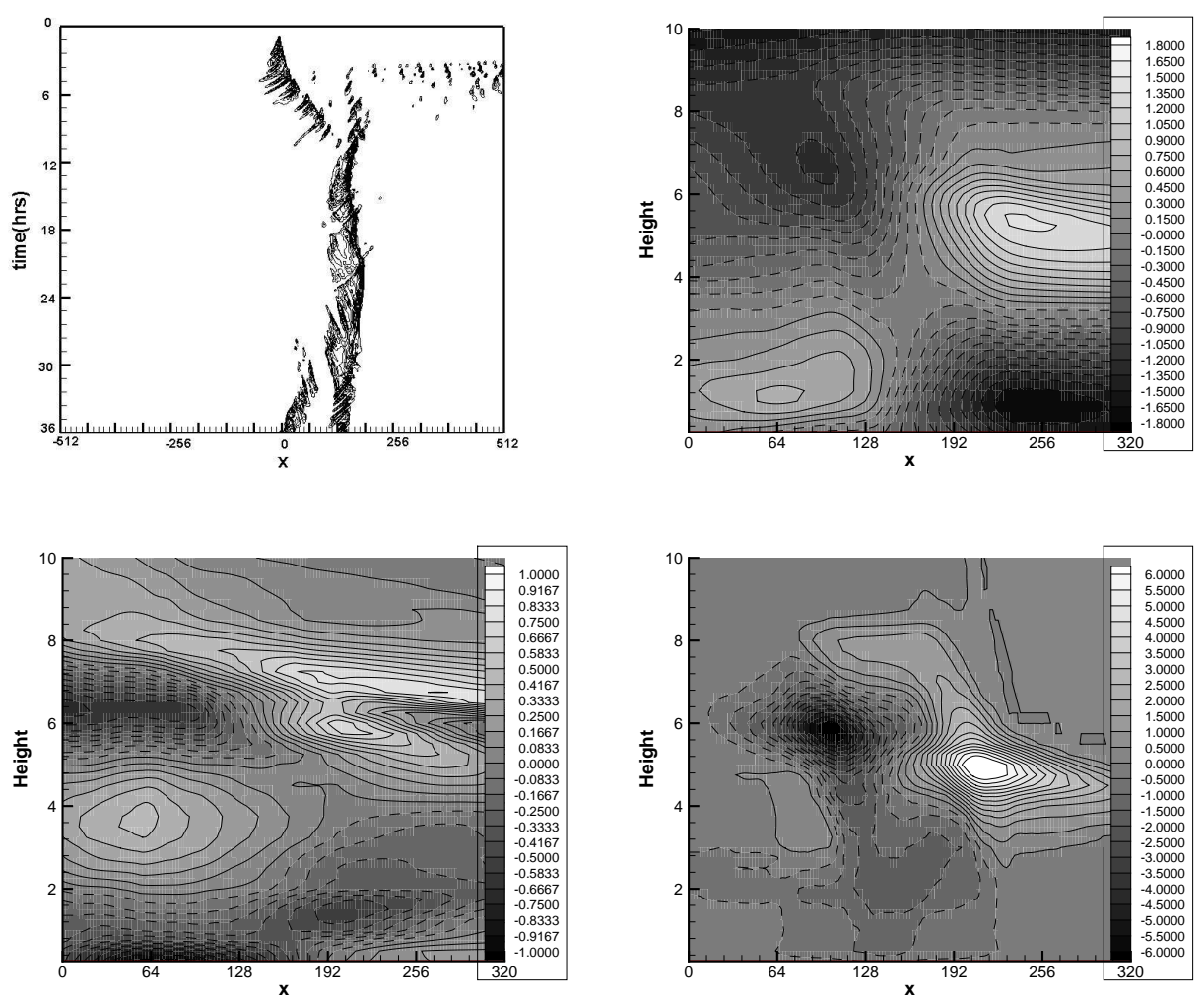

FIG. 2.5. Same as figure 2.4, but for $a=0.8$.

to provide a mathematical framework for clarifying these important issues.

\section{Derivation of the multi-scale model for squall lines}

With the background in section 2 about some basic physical effects in squall lines, we use asymptotic analysis to develop new systematic Multi-scale Squall Line Models (MSSLM) on mesoscales and microscales in this section.

3.1. General derivation in 3-D. We first repeat a simplified version of equation (2.2):

\section{Equations of motion}

$$
\begin{aligned}
& \frac{\mathrm{D} \mathbf{u}_{\mathrm{h}}}{\mathrm{D} t}=-\nabla_{h} p \\
& \frac{\mathrm{Dw}}{\mathrm{D} t}=-p_{z}+\epsilon^{-1}(\theta)-S_{w} \\
& \frac{\mathrm{D} \theta}{\mathrm{D} t}=-\epsilon^{-1} w+S_{\theta} \\
& \operatorname{div}_{h} \mathbf{u}_{h}+w_{z}=0,
\end{aligned}
$$

where the details for $S_{\theta}$ and $S_{w}$ can be found in equation (2.2). Equation (2.4) for the mixed ratios $q_{v}, q_{c}$ and $q_{r}$ are ignored here and are regarded as specifying the source terms $S_{\theta}, S_{w}$. Also, for simplicity in exposition a uniform vertical density $\rho(z)$ and 

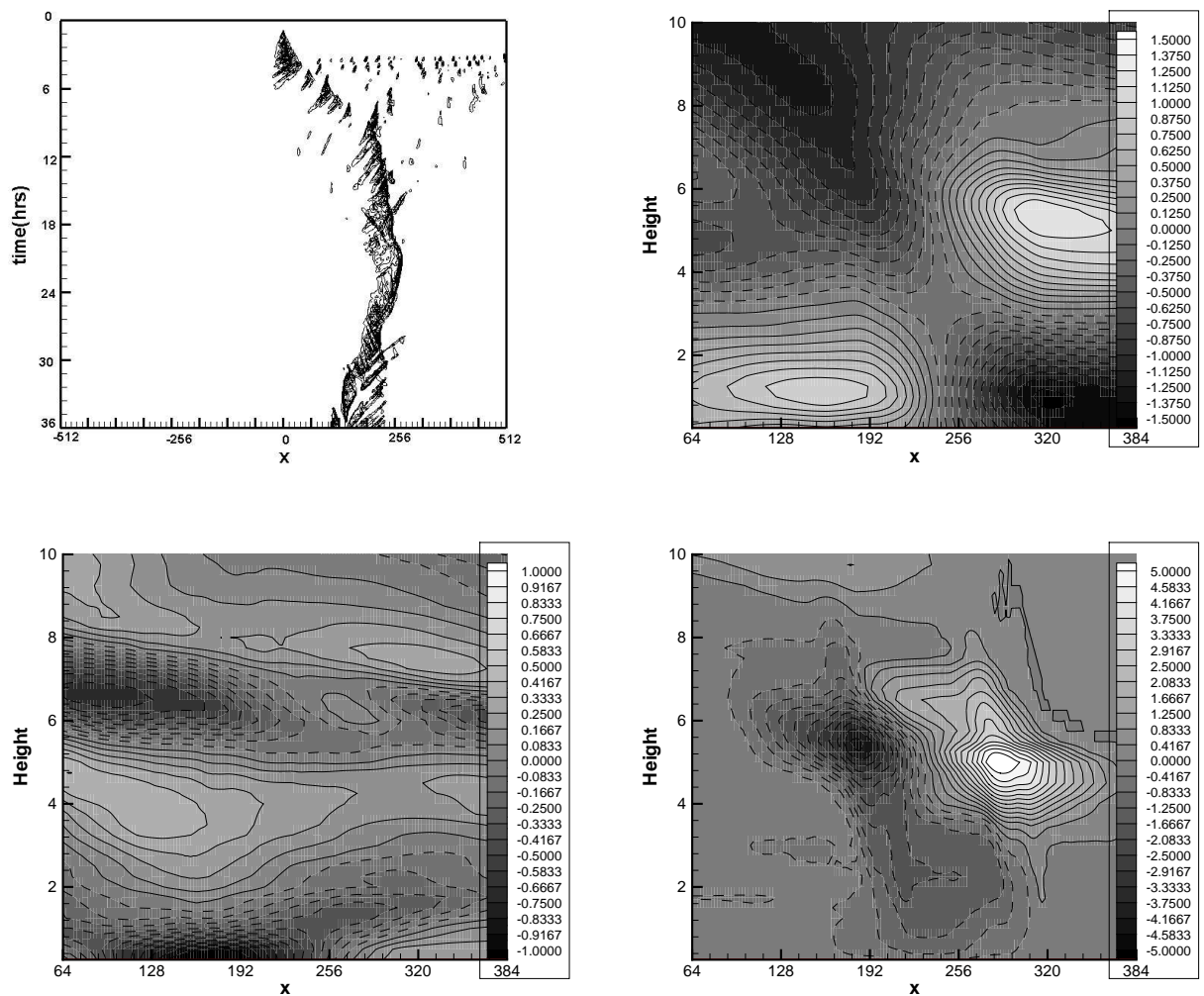

FIG. 2.6. Same as figure 2.4, but for $a=0.5$.

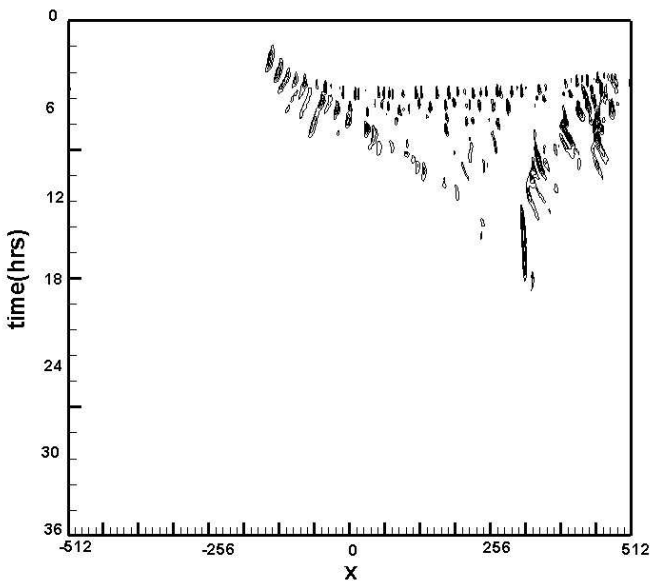

FIG. 2.7. The contours of surface precipitation from the dying scattered convection simulation with $a=0.3$ 

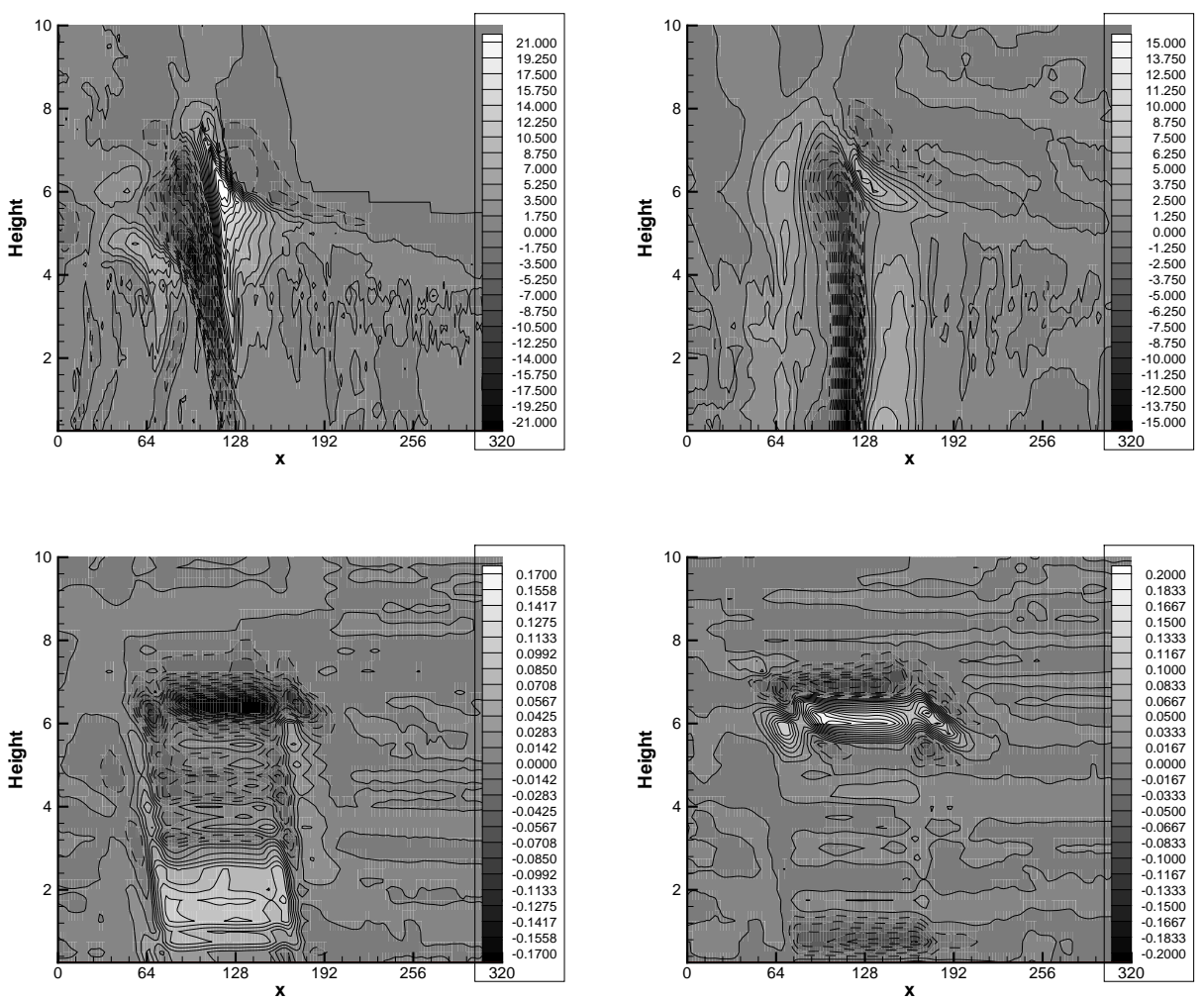

FIG. 2.8. The contours of the variables from the squall-line simulation with a=1. Top left: Source term fluctuation $\left\langle S_{\theta}^{\prime}\right\rangle$; Top right: Source term fluctuation $\left\langle S_{w}^{\prime}\right\rangle$; Bottom left: The eddy flux $\overline{\left(\left\langle u^{\prime}\right\rangle\left\langle w^{\prime}\right\rangle\right)_{z}}$; Bottom right: The eddy flux $\overline{\left(\left\langle w^{\prime}\right\rangle\left\langle\theta^{\prime}\right\rangle\right)_{z}}$. Note: 1, the units for the $x$-axis and height are both $\mathrm{km}$ in these plots, rather than the non-dimensional units; 2, a solid contour line represents positive value and a dashed one represents negative value.

constant $N^{2}(z)$ are assumed here. We focus on the spatial microscales

$$
L_{m}=10 \mathrm{~km} \quad \text { and } \quad T_{m}=1.5 \mathrm{~min},
$$

and spatial mesoscales

$$
L_{M}=\epsilon^{-1} L_{m}=100 \mathrm{~km} \quad \text { and } \quad T_{M}=\epsilon^{-1} T_{m}=15 \mathrm{~min},
$$

with the same nondimensional units (see (3.10) below) and $\epsilon \approx 0.1$ as in section 2.1. With these basic units, we expand the solutions of (3.1) as functions of $\mathbf{x}_{h}, t, \mathbf{X}_{h}=\epsilon \mathbf{x}_{h}$, and $\tau=t / \epsilon$ reflecting all the scales in (3.2) and (3.3). First, we recall some basic facts of multiple scales. Given a general function $f\left(\mathbf{X}_{h}, \mathbf{x}_{h}, t, \tau\right)$, the spatial and time average of $f$ over the microscales of order ten kilometers in space and order 1.5 minutes in time are defined by

$$
\begin{aligned}
\bar{f}\left(\mathbf{X}_{h}, t, \tau\right) & =\lim _{L \rightarrow \infty} \frac{1}{(2 L)^{2}} \int_{-L}^{L} \int_{-L}^{L} f d x d y \\
\langle f\rangle\left(\mathbf{X}_{h}, \mathbf{x}_{h}, t\right) & =\lim _{\tilde{T} \rightarrow \infty} \frac{1}{2 \tilde{T}} \int_{-\tilde{T}}^{\tilde{T}} f d \tau,
\end{aligned}
$$



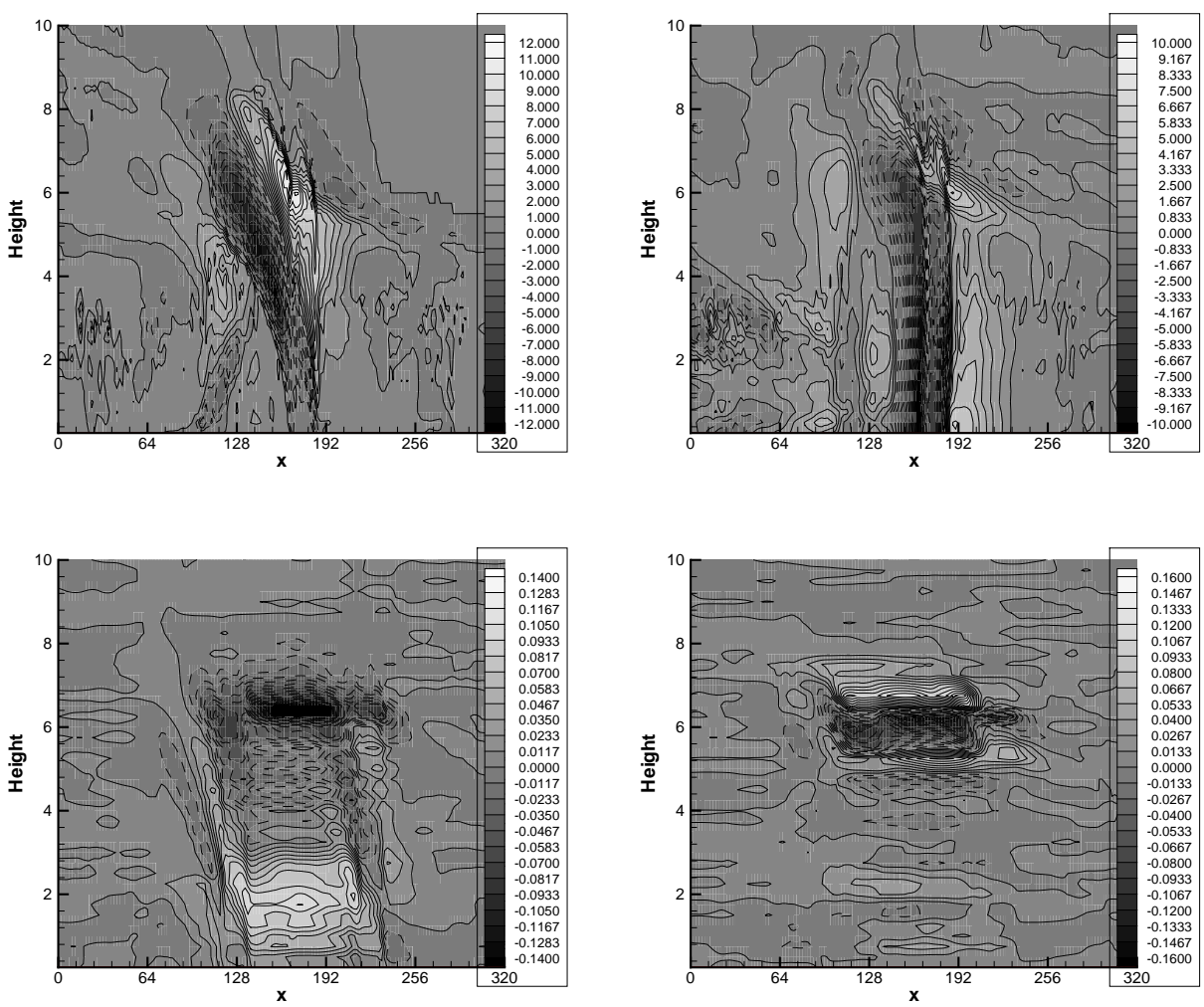

FIG. 2.9. Same as figure 2.8, but for $a=0.8$.

with the variables $\left(\mathbf{X}_{h}, t\right)$ and $\left(\mathbf{x}_{h}, \tau\right)$ regarded as independent variables. In the multiscale procedure, the space-time gradient of $f\left(\mathbf{X}_{h}, \mathbf{x}_{h}, t, \tau\right)$ is calculated according to the chain rule as

$$
\epsilon \nabla_{\mathbf{X}_{h}} f+\nabla_{\mathbf{x}_{h}} f, \quad \frac{\partial f}{\partial t}+\epsilon^{-1} \frac{\partial f}{\partial \tau} .
$$

For a function $f\left(\mathbf{X}_{h}, t, \tau\right)$ involving the mesoscale in space and the two time scales with time average $\langle f\rangle\left(\mathbf{X}_{h}, t\right)$, we define the fast time scale fluctuations by

$$
\tilde{f}\left(\mathbf{X}_{h}, t, \tau\right)=f-\langle f\rangle .
$$

A general function of both spatio-temporal scales, $f\left(\mathbf{X}_{h}, \mathbf{x}_{h}, t, \tau\right)$, has a large scale spatial mean, $\bar{f}\left(\mathbf{X}_{h}, t, \tau\right)$, so that

$$
f\left(\mathbf{X}_{h}, \mathbf{x}_{h}, t, \tau\right)=\bar{f}\left(\mathbf{X}_{h}, t, \tau\right)+f^{\prime}\left(\mathbf{X}_{h}, \mathbf{x}_{h}, t, \tau\right),
$$

where the fluctuations, $f^{\prime}$, automatically satisfy $\overline{f^{\prime}} \equiv 0$. With the above definitions, a general function $f\left(\mathbf{X}_{h}, \mathbf{x}_{h}, t, \tau\right)$ has the decomposition

$$
f\left(\mathbf{X}_{h}, \mathbf{x}_{h}, t, \tau\right)=\langle\bar{f}\rangle\left(\mathbf{X}_{h}, t\right)+\tilde{\bar{f}}\left(\mathbf{X}_{h}, t, \tau\right)+f^{\prime}\left(\mathbf{X}_{h}, \mathbf{x}_{h}, t, \tau\right),
$$

into the space-time average plus temporal fluctuations of the spatial mean plus spatialtemporal fluctuations. 

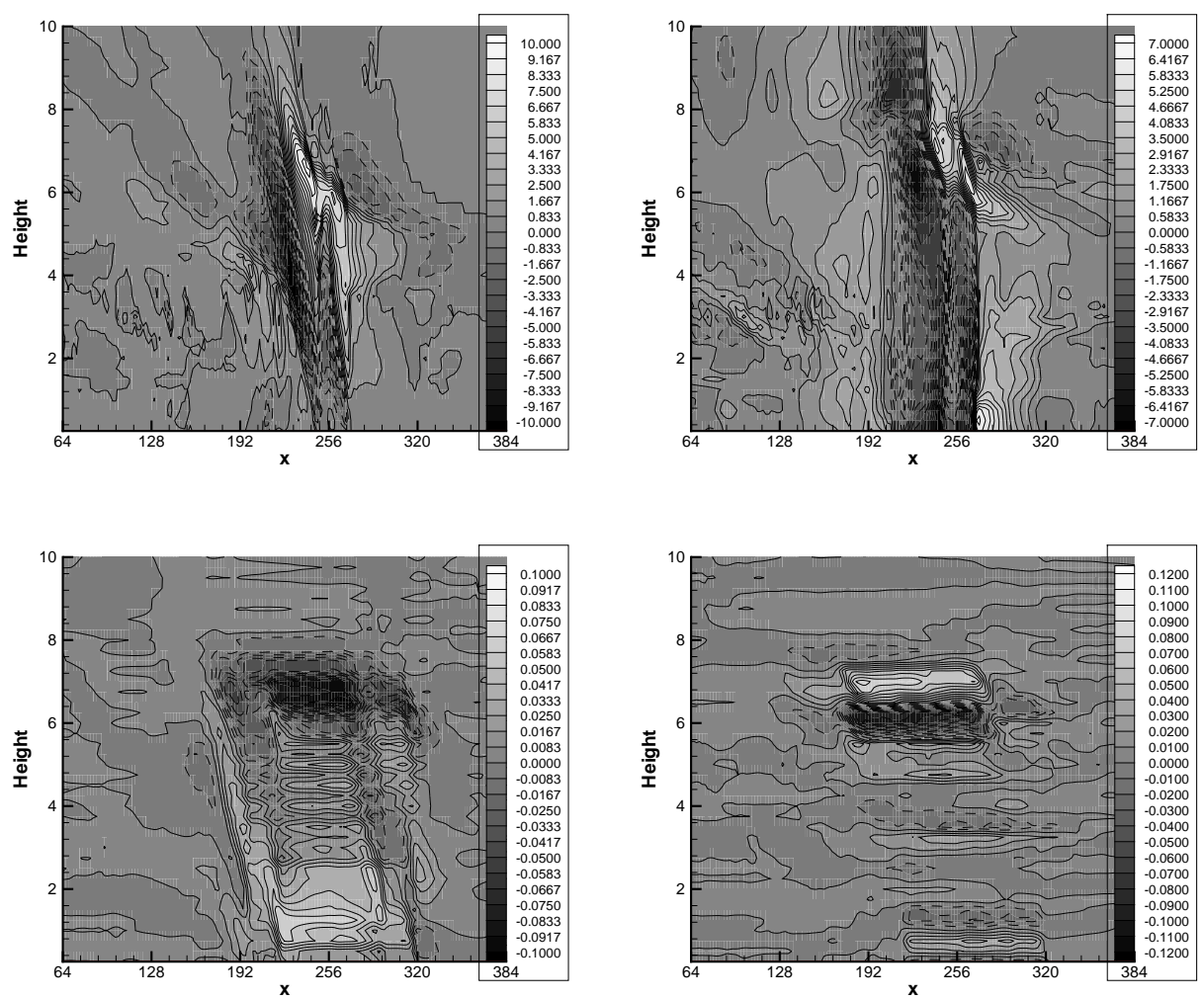

Fig. 2.10. Same as figure 2.8, but for $a=0.5$.

With the above motivation and definitions, we start with the following general ansatz to develop the multi-scale squall line models,

$$
\begin{aligned}
& \mathbf{u}_{h}=\epsilon^{-1} \mathbf{U}_{h}\left(\mathbf{X}_{h}, z, t\right)+\left(\mathbf{u}_{h}^{\prime}+\overline{\mathbf{u}}_{h}\right)+\epsilon \mathbf{u}_{h, 1} \\
& w=W\left(\mathbf{X}_{h}, z, t\right)+\tilde{\bar{w}}+w^{\prime}+\epsilon\left(\bar{w}+w_{1}^{\prime}\right)+\epsilon^{2}\left(\bar{w}_{2}+w_{2}^{\prime}\right) \\
& p=\epsilon^{-2} P\left(\mathbf{X}_{h}, z, t\right)+\epsilon^{-1}\left(\bar{p}_{-1}+p_{-1}^{\prime}\right) \\
& \theta=\epsilon^{-1} \Theta\left(\mathbf{X}_{h}, z, t\right)+\left(\theta^{\prime}+\bar{\theta}\right)+\epsilon \theta_{1} .
\end{aligned}
$$

In (3.8), we have used capital letters like $\mathbf{U}_{h}, W$, etc to denote the leading order space-time average over the small fast scales, i.e. $\mathbf{U}_{h}=\left\langle\overline{\mathbf{u}}_{h,-1}\right\rangle$, etc. We also assume the following expansions for the source terms

$$
\begin{aligned}
& S_{\theta}=\epsilon^{-1}\left(S_{\theta,-1}^{\prime}+\bar{S}_{\theta,-1}\right)+\left(S_{\theta, 0}^{\prime}+\bar{S}_{\theta, 0}\right) \\
& S_{w}=\epsilon^{-1}\left(S_{w,-1}^{\prime}+\bar{S}_{w,-1}\right)+\left(S_{w, 0}^{\prime}+\bar{S}_{w, 0}\right) .
\end{aligned}
$$

In (3.8), we use the variables with the following scales which include those in section 


$$
\begin{aligned}
& {[t]=15 \min , \quad[\tau]=1.5 \min , \quad \tau=\frac{t}{\epsilon},} \\
& {\left[\mathbf{x}_{h}\right]=10 \mathrm{~km}, \quad\left[\mathbf{X}_{h}\right]=100 \mathrm{~km}, \quad \mathbf{X}_{h}=\epsilon \mathbf{x}_{h},} \\
& {\left[\mathbf{u}_{h}\right]=[w]=10 \mathrm{~m} / \mathrm{s}, \quad[\theta]=3 \mathrm{~K},} \\
& {\left[S_{\theta}\right]=30 \mathrm{~K} / 15 \mathrm{~min}, \quad\left[S_{w}\right]=100 \mathrm{~m} / \mathrm{s} / 15 \mathrm{~min} .}
\end{aligned}
$$

The main new assumption here, compared with earlier work [26, 31], is that there exists a possibly strong large scale zonal flow $\epsilon^{-1} \mathbf{U}_{h}$. We have already seen the importance of large scale shears in squall line dynamics in section 2. So this assumption is natural here.

a. Strong large scale background flow. After the substitution of the ansatz (3.8) and (3.9) into the equation (3.1), a spatial and temporal average over the microscale scales is taken to develop the model on the mesoscale. We then collect the terms of different orders, with $\frac{\mathrm{D}}{\mathrm{D} t}=\frac{\partial}{\partial t}+\mathbf{U}_{h} \cdot \nabla_{\mathbf{X}_{h}}+W \frac{\partial}{\partial z}$ denoting the large scale average advection. Order $\epsilon^{-1}$ of the space-time averaged momentum equation gives us

$$
\frac{\mathrm{D} \mathbf{U}_{\mathrm{h}}}{\mathrm{D} t}=-\nabla_{\mathbf{x}_{h}} P
$$

We also pick the leading order $\epsilon^{-2}, \epsilon^{-1}$ and $\epsilon^{0}$ of the space-time averaged vertical momentum equation, potential temperature equation, and conservation of mass respectively

$$
\begin{aligned}
\frac{\partial}{\partial z} P & =\Theta \\
\frac{\mathrm{D} \Theta}{\mathrm{D} t} & =-W+\left\langle\bar{S}_{\theta,-1}\right\rangle \\
\operatorname{div}_{\mathbf{X}_{h} \mathbf{U}_{h}+W_{z}} & =0 .
\end{aligned}
$$

The equations above in (3.11) and (3.12) are simply the nonlinear Boussinesq equations with hydrostatic balance on $100 \mathrm{~km}$ scales. They apply if the forcing satisfies

$$
\left\langle\bar{S}_{\theta,-1}\right\rangle \neq 0,
$$

i.e., if there is heating of $120 \mathrm{~K} / \mathrm{hr}$ on the scale of order $100 \mathrm{~km}$, and $W \equiv\langle\bar{w}\rangle$ has velocities of $10 \mathrm{~m} / \mathrm{s}$; this actually does not happen in nature. Examples illustrate a weaker $\left\langle\bar{S}_{\theta,-1}\right\rangle$, as shown in figure 2.4, 2.5 and 2.6. In general, we make

Assumption 3.1. $\left\langle\bar{S}_{\theta,-1}\right\rangle=0$.

It is important to note that we can find special solutions of the above equation (3.11) and (3.12) satisfying Assumption 3.1, with $\Theta \equiv z$ the background stratification, $W \equiv 0$ and $\mathbf{U}_{h}=(U(z), V(z))$; these are shear flows and provide the background flow in squall lines. There are even more general time-dependent solutions of this type with $P \equiv 0$.

b. Fast-time equations. First we study the small scale fluctuation on the fast time scale. We substitute the ansatz (3.8) and (3.9) into the equation (3.1) and then compute the corresponding fast-time fluctuation equations. We collect the order $\epsilon^{-1}$ 
of the momentum, temperature equations and order $\epsilon^{0}$ of conservation of mass

$$
\begin{aligned}
\frac{\partial \mathbf{u}_{h}^{\prime}}{\partial \tau}+\mathbf{U}_{h} \cdot \nabla_{\mathbf{x}_{\mathbf{h}}} \mathbf{u}_{h}^{\prime}+w^{\prime}\left(\mathbf{U}_{h}\right)_{z} & =-\nabla_{\mathbf{x}_{\mathbf{h}}} p_{-1}^{\prime} \\
\frac{\partial \theta^{\prime}}{\partial \tau}+\mathbf{U}_{h} \cdot \nabla_{\mathbf{x}_{\mathbf{h}}} \theta^{\prime}+w^{\prime} \Theta_{z} & =-w^{\prime}+S_{\theta,-1}^{\prime} \\
\frac{\partial w^{\prime}}{\partial \tau}+\mathbf{U}_{h} \cdot \nabla_{\mathbf{x}_{\mathbf{h}}} w^{\prime} & =-\left(p_{-1}^{\prime}\right)_{z}-S_{w,-1}^{\prime}+\theta^{\prime} \\
\operatorname{div}_{\mathbf{x}_{\mathbf{h}}} \mathbf{u}_{h}^{\prime}+w_{z}^{\prime} & =0 .
\end{aligned}
$$

The equations in (3.13) with $\Theta \equiv z$ generalize the non-hydrostatic time dependent Taylor-Goldstein equations [18] from 2-D, i.e., for the special case with $\mathbf{U}_{h}=(U(z), 0)$.

Next, we study the large scale fluctuations on the fast time scale, $\tilde{\overline{\mathbf{u}}}_{h}, \tilde{\bar{w}}, \tilde{\bar{\theta}}$. From order $\epsilon^{0}$ of conservation of mass, it is easy to show that $\tilde{\bar{w}}_{z}=0$, so the non-slip boundary condition $\left.\tilde{\bar{w}}\right|_{z=0}=0$ guarantees $\tilde{\bar{w}} \equiv 0$. With $\tilde{\bar{w}} \equiv 0$, the large scale fasttime fluctuations in the order $\epsilon^{-1}$ momentum equations and potential temperature equation are

$$
\begin{aligned}
\frac{\partial \tilde{\bar{u}}_{h}}{\partial \tau} & =0 \\
\frac{\partial \tilde{\bar{\theta}}}{\partial \tau} & =\tilde{\bar{S}}_{\theta,-1} \\
0 & =-\left(\tilde{\bar{p}}_{-1}\right)_{z}-\tilde{\bar{S}}_{w,-1}+\tilde{\bar{\theta}} .
\end{aligned}
$$

The first equation shows that $\tilde{\bar{u}}_{h}=\tilde{\bar{u}}_{h}^{0}\left(\mathbf{X}_{\mathbf{h}}, z, t\right)$, and by definition $\langle\tilde{f}\rangle \equiv 0$ so $\tilde{\bar{u}}_{h}^{0} \equiv 0$. The last two equations in (3.14) define the large scale fast time fluctuations of potential temperature and pressure. As shown in the examples from section 2, in general the large scale fluctuating heat sources and vertical momentum sources are weak, $\tilde{\bar{S}}_{\theta,-1}, \tilde{\bar{S}}_{w,-1} \equiv 0$ and all large scale fast-time fluctuations vanish; if they are nonzero mathematically, then their vertical averages need to satisfy simple compatibility conditions.

c. Large scale equations. So far we have assessed the perturbation contributions to the strong large scale background flow from the smaller scale fast time fluctuations. Next we calculate the large scale space-time averaged perturbation field. Thus, we collect the order $\epsilon^{0}, \epsilon^{-1}, \epsilon^{0}$ and $\epsilon^{1}$ of the space-time averaged horizontal momentum equation, the vertical momentum equation, potential temperature equation and conservation of mass, respectively. With the definitions, $\left\langle\overline{\mathbf{u}}_{h}\right\rangle=\mathbf{u}_{h},\langle\bar{\theta}\rangle=\theta$, $\langle\bar{w}\rangle=w,\langle\bar{p}\rangle=p,\left\langle\bar{S}_{\theta, 0}\right\rangle=S_{\theta, 0},\left\langle\bar{S}_{w,-1}\right\rangle=S_{w,-1}$ and $\frac{\mathrm{D}}{\mathrm{D} t}=\frac{\partial}{\partial t}+\mathbf{U}_{h} \cdot \nabla_{\mathbf{X}_{h}}+W \frac{\partial}{\partial z}$, we obtain the Large Scale Equations

$$
\begin{aligned}
\frac{\mathrm{D}}{\mathrm{D} t} \mathbf{u}_{h}+w\left(\mathbf{U}_{h}\right)_{z} & =-p_{\mathbf{X}_{h}}-\left\langle\overline{w^{\prime} \mathbf{u}_{h}^{\prime}}\right\rangle_{z} \\
p_{z} & =\theta-S_{w,-1} \\
\frac{\mathrm{D}}{\mathrm{D} t} \theta+\mathbf{u}_{h} \cdot \nabla_{\mathbf{X}_{h}} \Theta+w \cdot \frac{\partial}{\partial z} \Theta & =S_{\theta, 0}-\left\langle\overline{w^{\prime} \theta^{\prime}}\right\rangle_{z} \\
\operatorname{div}_{\mathbf{X}_{h}} \mathbf{u}_{h}+w_{z} & =0 .
\end{aligned}
$$

Note that since the large scale fast-time vertical velocity fluctuations, $\tilde{\bar{w}}$, satisfy $\tilde{\bar{w}} \equiv 0$, the large scale fast-time contributions to the turbulent fluxes in (3.15) always 
vanish. However, the large scale perturbation equations in (3.15) are driven both by mean source terms and turbulent eddy flux divergence from the fluctuations. Next, we address how these turbulent fluxes are calculated from the fluctuating equations.

For the upscale eddy fluxes in (3.15), we write

$$
\begin{aligned}
\mathbf{u}_{h}^{\prime}=\left\langle\mathbf{u}_{h}^{\prime}\right\rangle+\mathbf{u}_{h}^{\prime \prime}, & \left\langle\mathbf{u}_{h}^{\prime \prime}\right\rangle=0 \\
w^{\prime}=\left\langle w^{\prime}\right\rangle+w^{\prime \prime}, & \left\langle w^{\prime \prime}\right\rangle=0, \\
\theta^{\prime}=\left\langle\theta^{\prime}\right\rangle+\theta^{\prime \prime}, & \left\langle\theta^{\prime \prime}\right\rangle=0 .
\end{aligned}
$$

Then, we have

$$
\begin{aligned}
\left\langle\overline{\mathbf{u}_{h}^{\prime} w^{\prime}}\right\rangle & =\overline{\left\langle\mathbf{u}_{h}^{\prime}\right\rangle\left\langle w^{\prime}\right\rangle}+\left\langle\overline{\mathbf{u}_{h}^{\prime \prime} w^{\prime \prime}}\right\rangle, \\
\left\langle\overline{\theta^{\prime} w^{\prime}}\right\rangle & =\overline{\left\langle\theta^{\prime}\right\rangle\left\langle w^{\prime}\right\rangle}+\left\langle\overline{\theta^{\prime \prime} w^{\prime \prime}}\right\rangle,
\end{aligned}
$$

where the right hand sides $\overline{\left\langle\theta^{\prime}\right\rangle\left\langle w^{\prime}\right\rangle}$ and $\overline{\left\langle\mathbf{u}_{h}^{\prime}\right\rangle\left\langle w^{\prime}\right\rangle}$ are the stationary turbulent eddy fluxes and the terms $\left\langle\overline{\mathbf{u}_{h}^{\prime \prime} w^{\prime \prime}}\right\rangle$ and $\left\langle\overline{\theta^{\prime \prime} w^{\prime \prime}}\right\rangle$ are the transient eddy fluxes. In general, for squall lines the stationary turbulent fluxes in the traveling wave reference frame are more significant than the transient ones. Then we can make

ASSUMPTION 3.2. For propagating traveling waves, only the stationary turbulent eddy flux in the traveling reference frame is important.

With the above simplifying assumption, we just need to model the stationary eddy fluxes in the large scale model. There is a simple way to obtain closed equations for the stationary eddy fluxes. Take equation (3.13) for the small-scale fluctuations and average in the fast time to obtain generalized steady Taylor-Goldstein equations

$$
\begin{aligned}
\mathbf{U}_{h} \cdot \nabla_{\mathbf{x}_{\mathbf{h}}}\left\langle\mathbf{u}_{h}^{\prime}\right\rangle+\left\langle w^{\prime}\right\rangle\left(\mathbf{U}_{h}\right)_{z} & =-\nabla_{\mathbf{x}_{\mathbf{h}}}\left\langle p_{-1}^{\prime}\right\rangle \\
\mathbf{U}_{h} \cdot \nabla_{\mathbf{x}_{\mathbf{h}}}\left\langle\theta^{\prime}\right\rangle+\left\langle w^{\prime}\right\rangle \Theta_{z} & =\left\langle S_{\theta,-1}^{\prime}\right\rangle \\
\mathbf{U}_{h} \cdot \nabla_{\mathbf{x}_{\mathbf{h}}}\left\langle w^{\prime}\right\rangle & =-\left\langle p_{-1}^{\prime}\right\rangle_{z}-\left\langle S_{w,-1}^{\prime}\right\rangle+\left\langle\theta^{\prime}\right\rangle \\
\operatorname{div}_{\mathbf{x}_{\mathbf{h}}}\left\langle\mathbf{u}_{h}^{\prime}\right\rangle+\left\langle w^{\prime}\right\rangle_{z} & =0 .
\end{aligned}
$$

Thus, with Assumption 3.2, the solution of the steady equations in (3.18) can be found and utilized to compute $\overline{\left\langle\theta^{\prime}\right\rangle\left\langle w^{\prime}\right\rangle}$ and $\overline{\left\langle\mathbf{u}_{h}^{\prime}\right\rangle\left\langle w^{\prime}\right\rangle}$ as needed in the stationary eddy fluxes. An example is illustrated in section 4 .

3.2. Specialization to 2-D. In this subsection, we specialize the $3-\mathrm{D}$ multiscale squall line model (3.15) and (3.13) to the $2-\mathrm{D}$ case, since the $2 \mathrm{D}$ version is much simpler and already contains the main feature of the squall line. We assume $\mathbf{U}_{h}=$ $(\bar{U}(z), 0), \Theta=z$, and everything is a function of $\mathrm{x}$ and $\mathrm{z}$ only. The direct simplification gives us the following equations.

A) Small Scales

$$
\begin{aligned}
u_{\tau}^{\prime}+\bar{U} u_{x}^{\prime}+w^{\prime} \bar{U}_{z} & =-\left(p_{-1}^{\prime}\right)_{x} \\
w_{\tau}^{\prime}+\bar{U} w_{x}^{\prime} & =-\left(p_{-1}^{\prime}\right)_{z}+\theta^{\prime}-S_{w,-1}^{\prime} \\
\theta_{\tau}^{\prime}+\bar{U} \theta_{x}^{\prime} & =-w^{\prime}+S_{\theta,-1}^{\prime} \\
u_{x}^{\prime}+w_{z}^{\prime} & =0 \quad \overline{u^{\prime}, \bar{w}^{\prime}, \bar{\theta}^{\prime}=0 .}
\end{aligned}
$$


B) Large Scales

$$
\begin{aligned}
\frac{D}{D t} u+w \bar{U}_{z} & =-p_{X}-{\overline{\left\langle w^{\prime} u^{\prime}\right\rangle}}_{z} \\
p_{z} & =\theta-\bar{S}_{w,-1} \\
\frac{D}{D t} \theta & =-w+\bar{S}_{\theta, 0}-\overline{\left\langle w^{\prime} \theta^{\prime}\right\rangle_{z}} \\
u_{X}+w_{z} & =0
\end{aligned}
$$

with $\frac{D}{D t}=\frac{\partial}{\partial t}+\bar{U}(z) \frac{\partial}{\partial X}$.

Note that the small scale equation is now a forced non-hydrostatic time-dependent Taylor-Goldstein Equation. Also, the large scale equation in (3.20) is a forced hydrostatic Taylor-Goldstein equation with essentially the same structure but forced by turbulent fluxes from the small scales. These equations display yet another example of self-similarity across scales in stratified flows [32]. The steady state solution of the small scale equations gives us the necessary information for the large scale models under Assumptions 3.1 and 3.2, which we assume here. In section 4, we illustrate how to solve these equations numerically.

3.3. Comparison with simpler multi-scale models. In [26], Klein and Majda studied scale interaction in the atmosphere involving moist physics, and developed multi-scale models for mesoscale organized convection. Two spatial scales (the same as ours here) and the single time scale $([t]=15 \mathrm{~min})$ are explored there. Repeating those ideas, but replacing the active moisture effects by source terms as in (3.9), the following multi-scale models are obtained under the weak temperature gradient (WTG) and low Froude number assumptions. (A similar ansatz is used without the large scale zonal shear $\epsilon^{-1} \mathbf{U}_{h}, \epsilon^{-1} \Theta$, etc as in (3.8) and (3.9).)

Small Scales

$$
\begin{aligned}
\theta^{\prime} & =S_{w,-1}^{\prime} \\
w^{\prime} & =S_{\theta,-1}^{\prime} \\
\frac{D \mathbf{u}_{h}^{\prime}}{D t} & =-\nabla_{h} p^{\prime}+\left(\overline{w^{\prime} \mathbf{u}_{h}^{\prime}}\right)_{z} \\
\operatorname{div}_{\mathbf{x}_{\mathbf{h}}} \mathbf{u}_{h}^{\prime}+w_{z}^{\prime} & =0 .
\end{aligned}
$$

Large Scales

$$
\begin{aligned}
\frac{\partial \overline{\mathbf{u}}_{h}}{\partial t} & =-\nabla \mathbf{x} \bar{p}-\left(\overline{w^{\prime} \mathbf{u}_{h}^{\prime}}\right)_{z} \\
\bar{p}_{z} & =\bar{\theta}-\bar{S}_{w,-1} \\
\frac{\partial \bar{\theta}}{\partial t} & =-\bar{w}+\bar{S}_{\theta, 0}-\left(\overline{w^{\prime} \theta^{\prime}}\right)_{z} \\
\operatorname{div}_{\mathbf{X}_{\mathbf{h}}} \overline{\mathbf{u}}_{h}+\bar{w}_{z} & =0 .
\end{aligned}
$$

In (3.21), the advective derivative is given by $\frac{D}{D t}=\frac{\partial}{\partial t}+\left(\overline{\mathbf{u}}_{h}+\mathbf{u}_{h}^{\prime}\right) \cdot \nabla_{\mathbf{x}_{\mathbf{h}}}+w^{\prime} \frac{\partial}{\partial z}$.

Comparing the large scale equations (3.22) with our large scale model (3.15), we notice that if we assume $\mathbf{U}_{h}=0$ and $\Theta=z$, those two models are exactly the same. Because there are no $\mathbf{U}_{h}$ and $\Theta$ in the ansatz used to derive these models, the above assumption is natural. Hence we can view our large scale model (3.15) as an extension of (3.22). The small scale models (3.21) and (3.13) are a little different, as these are 
derived with different time scales. But there are also some connections between them. Also we first assume $\mathbf{U}_{h}=0$ and $\Theta=z$ in (3.13). Then the first and second equations of (3.21) are identical to the steady solution in (3.18) with $p_{-1}^{\prime} \equiv 0$. The momentum equation has a different form due to the different time scale used. However, for a two-dimensional flow field $\mathbf{u}_{h}^{\prime}=\left(u^{\prime}(x, z, t), 0\right)$ the horizontal momentum equation in (3.21) is trivially satisfied and the equations in (3.21), (3.22) are exactly the limit of the large scale 2-D steady state equations in section 3.2 with $\bar{U} \equiv 0$ and $\Theta \equiv z$.

Consider the reduced asymptotic equations in (3.21) and (3.22) in 2-D; they have the advantage that they are readily solved explicitly for $\left(u^{\prime}(x, z, t), w^{\prime}(x, z, t)\right)$, so that the nonlinear momentum equation in (3.21) is trivially satisfied $[2,32,37]$. Earlier studies of convective momentum transport (CMT) have emphasized the importance of wave tilts for upscale momentum transport in squall lines [39, 40]. In this small scale model, there is a balance $\left(w^{\prime}=S_{\theta,-1}^{\prime}\right)$ between the vertical velocity $w^{\prime}$ and the potential temperature source $S_{\theta,-1}^{\prime}$. For the two-dimensional (x-z) setup, we consider a heat source with two phase-lagged baroclinic modes $S_{\theta,-1}^{\prime}=$ $k \cos [k x-w t] \sqrt{2} \sin (z)+\alpha k \cos \left[k\left(x+x_{0}\right)-\omega t\right] \sqrt{2} \sin (2 z)$ as a simple model for a tilted wave. Two key parameters here are $\alpha$, the strength of the second baroclinic heating, and $x_{0}$, the lag between the heating in the two vertical modes. We can then compute the vertical and zonal velocities of the small scale solution of (3.21) by the WTG balance $w^{\prime}=S_{\theta,-1}^{\prime}$ and the continuity equation $u_{x}^{\prime}+w_{z}^{\prime}=0$ :

$$
\begin{aligned}
& u^{\prime}(x, z, t)=-\sin [k x-w t] \sqrt{2} \cos (z)-2 \alpha \sin \left[k\left(x+x_{0}\right)-\omega t\right] \sqrt{2} \cos (2 z) \\
& w^{\prime}(x, z, t)=k \cos [k x-w t] \sqrt{2} \sin (z)+\alpha k \cos \left[k\left(x+x_{0}\right)-\omega t\right] \sqrt{2} \sin (2 z) .
\end{aligned}
$$

Then, the eddy flux divergence is given by

$$
\left\langle\overline{w^{\prime} u^{\prime}}\right\rangle_{z}=\frac{3}{2} \alpha k \sin \left(k x_{0}\right)[\cos (z)-\cos (3 z)] .
$$

Note that a third vertical baroclinic mode is generated. If either $\alpha$ or $x_{0}$ is zero, no eddy flux is generated. Hence both phase lags and wave tilts are important for the upscale momentum transport. Here in our new multi-scale squall line models, as we will see soon in section 4 , there is no such requirement of wave tilts to obtain upscale momentum transport. Heating alone plus shear creates tilt and upscale transports automatically.

The large scale equations in (3.22) are the forced hydrostatic linear gravity wave equations. These equations can be readily solved explicitly by vertical separation of variables [30] for the known source terms and small scale turbulent fluxes. In the present 2-D setting, the small scale model described above provides an elementary model for calculating the turbulent fluxes. However, despite their simplicity, these large scale equations fail to capture the advection by the large scale flow, unlike the new models developed here.

\section{Upscale transports in squall lines}

Here we present mathematical formulations of the multi-scale squall line equations from section 3 which lead to simple numerical algorithms. We demonstrate that the large scale model can capture the main large scale effect of the squall line simulations presented in section 2 . We also show some simple numerical tests for the small scale model alone, which show that upright heating plus shear alone can create nonzero eddy fluxes without any small scale phase lagged heating. 
4.1. Vorticity stream formulation and numerical methods. First, we refer to the 2D MSSLM from section 3.2. For the small scale model (3.19), we first introduce a stream function

$$
\left(u^{\prime}, w^{\prime}\right)=\left(-\psi_{z}^{\prime}, \psi_{x}^{\prime}\right)
$$

and vorticity $\omega^{\prime}$

$$
\omega^{\prime} \equiv-u_{z}^{\prime}+w_{x}^{\prime}=\psi_{x x}^{\prime}+\psi_{z z}^{\prime}=\Delta \psi^{\prime} .
$$

Taking the curl of (3.19) results in the Vorticity Stream Form (VSF)

$$
\begin{aligned}
\omega_{\tau}^{\prime}+U \omega_{x}^{\prime}-w^{\prime} U_{z z} & =\theta_{x}^{\prime}-\left(S_{w,-1}^{\prime}\right)_{x} \\
\theta_{\tau}^{\prime}+U \theta_{x}^{\prime} & =-w^{\prime}+S_{\theta,-1}^{\prime} \\
\Delta \psi^{\prime}=\omega^{\prime} \quad\left(u^{\prime}, w^{\prime}\right) & =\left(-\psi_{z}^{\prime}, \psi_{x}^{\prime}\right),
\end{aligned}
$$

by using the fact $w_{x x}^{\prime}+w_{z z}^{\prime}=\Delta w^{\prime}=\Delta \psi_{x}^{\prime}=\omega_{x}$.

For simplicity, a periodic domain in the small scale variable $x$ is used. A natural approach to solve the small scale equation in (3.19) numerically is to solve (4.3) by separation of variables in $x$, i.e., $\omega^{\prime}, \theta^{\prime}, \psi^{\prime}$, and the source terms are expanded via

$$
f(x, z)=\sum_{|k| \leq N} \tilde{f}^{k}(z) e^{i k x}, \quad \mathrm{k} \text { is an integer. }
$$

Time-dependent decoupled single space dimensional, linear ODE's are obtained, which are easy to solve.

If we assume that

$$
\begin{gathered}
\omega^{\prime}(x, z, t)=\sum_{|k| \leq N} \tilde{\omega}^{k}(z, t) e^{i k x}, \quad \psi^{\prime}(x, z, t)=\sum_{|k| \leq N} \tilde{\psi}^{k}(z, t) e^{i k x}, \\
\theta^{\prime}(x, z, t)=\sum_{|k| \leq N} \tilde{\theta}^{k}(z, t) e^{i k x},
\end{gathered}
$$

and

$$
S_{w,-1}^{\prime}=\sum_{|k| \leq N} \tilde{S}_{w}^{k}(z, t) e^{i k x}, \quad S_{\theta,-1}^{\prime}=\sum_{|k| \leq N} \tilde{S}_{\theta}^{k}(z, t) e^{i k x},
$$

we have

$$
\begin{aligned}
\frac{\partial}{\partial t} \tilde{\omega}^{k}(z, t)+i k U \tilde{\omega}^{k}-i k \tilde{\psi}^{k} U_{z z} & =i k \tilde{\theta}^{k}-i k \tilde{S}_{w}^{k} \\
\frac{\partial}{\partial t} \tilde{\theta}^{k}(z, t)+i k U \tilde{\theta}^{k} & =-i k \tilde{\psi}^{k}+\tilde{S}_{\theta}^{k}
\end{aligned}
$$

$\tilde{\psi}^{k}$ is calculated by solving the elliptic equation

$$
\left(\tilde{\psi}^{k}(z, t)\right)_{z z}-k^{2} \tilde{\psi}^{k}(z, t)=\tilde{\omega}^{k},
$$

with given boundary conditions as discussed below. 
Equation (4.4) can then be easily solved for each wave number $k$. We then collect $\tilde{\omega}^{k}(z, T)$ and $\tilde{\theta}^{k}(z, T)$ at stopping time $T$ in Fourier space and transfer them back into physical space, where $u$ and $w$ can be computed based on them.

A periodic boundary condition is used in the horizontal direction, hence the separation of variables method can be used. In the $z$ direction, we assume $w^{\prime}=0$ on $z=0$, hence

$$
\left.w^{\prime}\right|_{z=0}=0 \Leftrightarrow \tilde{w}(0, t)=0 .
$$

On the other boundary, we use a far-field radiation boundary condition when $z \rightarrow \infty$. Following [1], we have the radiation boundary condition

$$
w_{z}^{\prime}+k w^{\prime}=o\left(\frac{1}{z}\right), \quad z \rightarrow \infty,
$$

for each wave number $k$. Hence, one possible boundary condition is

$$
\left(\frac{\partial}{\partial z}+k\right) w^{\prime}=0
$$

We refer to [1] for more details about these radiation boundary conditions.

A similar strategy is used for the large scale models in (3.20) from section 3.2. Because $\omega=-u_{z}+\epsilon w_{X}$, the term $-u_{z}$ is the leading order component of the vorticity equation. Thus as in the above, we take the equation for $u_{z}$

$$
\begin{aligned}
\frac{\partial}{\partial t} u_{z}+U(z) u_{X z}+w U_{z z} & =-p_{X z}-\left(\overline{w^{\prime} u^{\prime}}\right)_{z z} \\
p_{X z} & =\theta_{X}-\left(S_{w,-1}\right)_{X} \\
\frac{D}{D t} \theta & =-w+S_{\theta, 0}-\left(\overline{w^{\prime} \theta^{\prime}}\right)_{z} \\
u_{X}+w_{z} & =0,
\end{aligned}
$$

and eliminate the pressure to get Vorticity Stream Form (VSF)

$$
\begin{aligned}
\frac{\partial}{\partial t} u_{z}+U(z)\left(u_{z}\right)_{X}+w U_{z z} & =-\theta_{X}+\left(S_{w,-1}\right)_{X}-\left(\overline{w^{\prime} u^{\prime}}\right)_{z z} \\
\frac{\partial}{\partial t} \theta+U(z) \theta_{X} & =-w+S_{\theta, 0}-\left(\overline{w^{\prime} \theta^{\prime}}\right)_{z} \\
\left(u_{z}\right)_{X}+w_{z z} & =0 .
\end{aligned}
$$

Comparing these equations with the VSF for small scale models (4.3), we can find those two have something in common. Actually if we make the substitution $\omega=-u_{z}$ in (4.8), these two VSFs share the same left hand side, and the only difference comes from the source terms. A nice version of self-similarity [32] on mesoscales and microscales is observed for these equations.

Once again, with this formulation the large scale equations (3.20) can be solved easily by separation of variables in $X$ "spectrally" as below (4.3). We then have

$$
\begin{aligned}
\frac{\partial}{\partial t} \tilde{w}_{z z}^{k}(z, t)+i k U \tilde{w}_{z z}^{k}-i k \tilde{w}^{k} U_{z z} & =-k^{2} \tilde{\theta}^{k}+k^{2} \tilde{S}_{w}^{k}+i k\left(\widetilde{\overline{w^{\prime} u^{\prime}}}\right)_{z z}^{k} \\
\frac{\partial}{\partial t} \tilde{\theta}^{k}(z, t)+i k U \tilde{\theta}^{k} & \left.=-\tilde{w}^{k}+\tilde{S}_{\theta}^{k}-\widetilde{\left(\widetilde{w^{\prime} \theta^{\prime}}\right.}\right)_{z}^{k},
\end{aligned}
$$


where $\tilde{w}^{k}$ is calculated by solving the elliptic equation

$$
\left(\tilde{w}^{k}(z, t)\right)_{z z}=\tilde{w}_{z z}^{k}(z, t) .
$$

Consistent with the self-similarity we observed above, the spectral form of these equations has a similar structure, as we can observe from (4.3) and (4.9). The only difference comes from the definition of vorticity under two scales: $\omega=-u_{z}$ in the large scale model and $\omega=-u_{z}^{\prime}+w_{x}^{\prime}$ in the small scale one. Hence the elliptic equation (4.10) can be viewed as the one for small scale (4.5) with $k=0$. Similar boundary conditions are employed, with $k=0$ in equation (4.6). Therefore, the same numerical methods can be used to solve these equations.

4.2. Large scale effect of mean heating. We are solving the large scale model (4.8) only to mimic a squall line experiment. In this illustration, we show that this model can qualitatively capture some features of the large scale effect of the squall line. Since we are interested in only qualitative changes here, we solve (3.20) without the changes in density with height, which are utilized in section 2 .

The same background shear with $a=1$, as shown in (2.5), is used. Now we need four input variables in (4.8): the source term $S_{\theta, 0}, S_{w,-1}$, and the eddy fluxes $\overline{\left\langle w^{\prime} \theta^{\prime}\right\rangle_{z}}$ and $\overline{\left\langle w^{\prime} u^{\prime}\right\rangle_{z}}$. First, the source term $S_{w,-1}$ is much smaller and less important, compared with $S_{\theta, 0}$, since in acceleration units of $10 \mathrm{~m} / \mathrm{s}$ per 15 minutes, $S_{w}$ in figure 2.8, 2.9, 2.10, has to be order 100 for $S_{w,-1}$ to be significant. Also, for this particular background shear our numerical tests demonstrate that the eddy fluxes $\overline{\left\langle w^{\prime} \theta^{\prime}\right\rangle_{z}}$ and ${\overline{\left\langle w^{\prime} u^{\prime}\right\rangle_{z}}}_{z}$ are also relatively smaller than the source term $S_{\theta, 0}$. Hence, $S_{\theta, 0}$ is the dominant term among these. A simple numerical test is that we keep $S_{\theta, 0}$ and leave the other three source terms zero. Then we can analyze how much of the large scale flow in the squall line is contributed by the source term $S_{\theta, 0}$. To calculate the source term $S_{\theta, 0}$, we go back to the 2D CRM squall line test in section 2.3. In the steady regime of that experiment, we compute the large scale average of the source term $\bar{S}_{\theta}$ and take the time average $\left\langle\bar{S}_{\theta}\right\rangle$ of it over several hours. This $\left\langle\bar{S}_{\theta}\right\rangle$ from the experiment is the input of the large scale model from $S_{\theta, 0}$ in the large scale model from (3.20) for our simple test here.

In the numerical test of (3.20), the domain size is set to be $1024 \mathrm{~km}$ with $15 \mathrm{~km}$ in vertical direction. We take zero initial conditions for $u_{z}, \theta$, and run this experiment for a long time to an equilibrium state, with $2 \mathrm{~km}$ horizontal and $0.25 \mathrm{~km}$ vertical resolution. The numerical solution $u$ can then be obtained through $u_{z}$. It is shown in the top left of figure 4.1. Note that from ansatz (3.8), we know that the spatialtemporal average of the horizontal velocity is

$$
u_{\epsilon}=\epsilon^{-1} U+u,
$$

where we represent the left hand side by $u_{\epsilon}$ for clarification. What we show in figure 4.1 is actually the velocity perturbation $u$ on the right hand side. We want to compare this $u$ with the corresponding large scale velocity perturbation obtained through the 2D CRM simulation in section 2.3, which is shown in the top right of figure 4.1. Note that the velocity shown in the top right of figure 2.4 is the total velocity $u_{\epsilon}$ on the left hand side of (4.11), and the velocity shown in the top right of figure 4.1 is the large scale velocity perturbation $u=u_{\epsilon}-\epsilon^{-1} U$. We also repeat the experiment with weaker background shears: $a=0.8$ and 0.5 . Similarly, we collect $S_{\theta}$ from the corresponding CRM tests as in section 2.3. The numerical results are shown in the middle and bottom parts of figure 4.1 . 
Comparing the left three figures with the right three of figure 4.1, we can observe similar squall line structure by eye. We also notice that in the area far from the center of the squall line and near the boundary, the qualitative agreement between them is not very good. We believe this is due to the periodic boundary condition we used, where the "shock like" jump updraft is aliased to a periodic domain which is not all that large. In the 2D CRM simulation, an open lateral boundary condition is employed. From this reason, we cannot expect the same structure near the boundary. We concentrate on the area near the center of squall line, and compute the correlation between them. The horizontal domain from $-256 \mathrm{~km}$ to $256 \mathrm{~km}$ is considered, and the correlation between the large scale horizontal velocity $u$ of the 2D CRM simulation and this test is $0.7573,0.7034$ and 0.6346 for the three cases $a=1,0.8$ and 0.5 respectively. From these, we also conclude that this simple test captures some main large scale effects of this squall line despite ignoring density changes with height. Of course another simple test is to add models for the other three source terms and include density changes, then check if they create an improved correlation with the CRM solution. We leave this for further research. The interested reader should consult $[6$, 28] for other physically interesting solutions of the hydrostatic forced Taylor-Goldstein equations.

4.3. The small scale model and upscale transports. The small scale model (4.3) is solved in this subsection. We show that simple vertical heating alone plus shear creates nontrivial upscale transports automatically without any small scale tilts in the heating in contrast to the model in section 3.3.

We use the same large scale background shear with $a=1$, as shown in (2.5). One critical issue to set up such an experiment is how we define the source term $S_{\theta,-1}^{\prime}$ and $\left(S_{w,-1}^{\prime}\right)_{x}$. For simplicity, we assume that the source term $\left(S_{w,-1}^{\prime}\right)_{x}$ is zero in this test for the same reason explained before since it is much weaker anyway. The heating $S_{\theta,-1}^{\prime}$ is defined as

$$
S_{\theta,-1}^{\prime}=e^{i k(x-c t)} Q_{0}(z)
$$

where $k=\frac{2 \pi}{L}$ and $c=0$ is taken. Following the plots of $S_{\theta}^{\prime}$ shown in the squall line simulation (figure $2.8,2.9$ and 2.10 ), we define $Q_{0}(z)$ as

$$
Q_{0}(z)= \begin{cases}b \sin (\pi z / 0.4) & \text { if } z<0.4 \\ 0 & \text { otherwise }\end{cases}
$$

and $b=1$ is taken in this experiment. A plot for $Q_{0}(z)$ is shown in figure 4.2. We take a zero initial condition for $\omega^{\prime}, \theta^{\prime}$, and run this experiment for a long time to an equilibrium state. The domain size is taken to be $51.2 \mathrm{~km}$ with $15 \mathrm{~km}$ in vertical direction.

In figure 4.3, we show some numerical results obtained after the steady state is reached. We also repeat the test with different background shears $\mathrm{a}=0.8$ and 0.5 in (2.5). Note that the main connection between the small scale model in (3.19) and large scale model (3.20) is through the eddy flux terms $\overline{\left\langle w^{\prime} \theta^{\prime}\right\rangle_{z}}$ and $\overline{\left\langle w^{\prime} u^{\prime}\right\rangle_{z}}$. Hence we utilize Assumption 2 and plot the flux terms $\overline{\left\langle w^{\prime}\right\rangle\left\langle\theta^{\prime}\right\rangle}$ and $\overline{\left\langle w^{\prime}\right\rangle\left\langle u^{\prime}\right\rangle}$ for these three tests in figure 4.4 , where we can clearly observe that a tilt has been generated in these fluxes. As we mentioned in section 3.3, earlier studies emphasize the importance of wave tilts for upscale momentum transport, and now we find out that here the shear itself produces the tilt, which is the important effect passed to the large scale model 

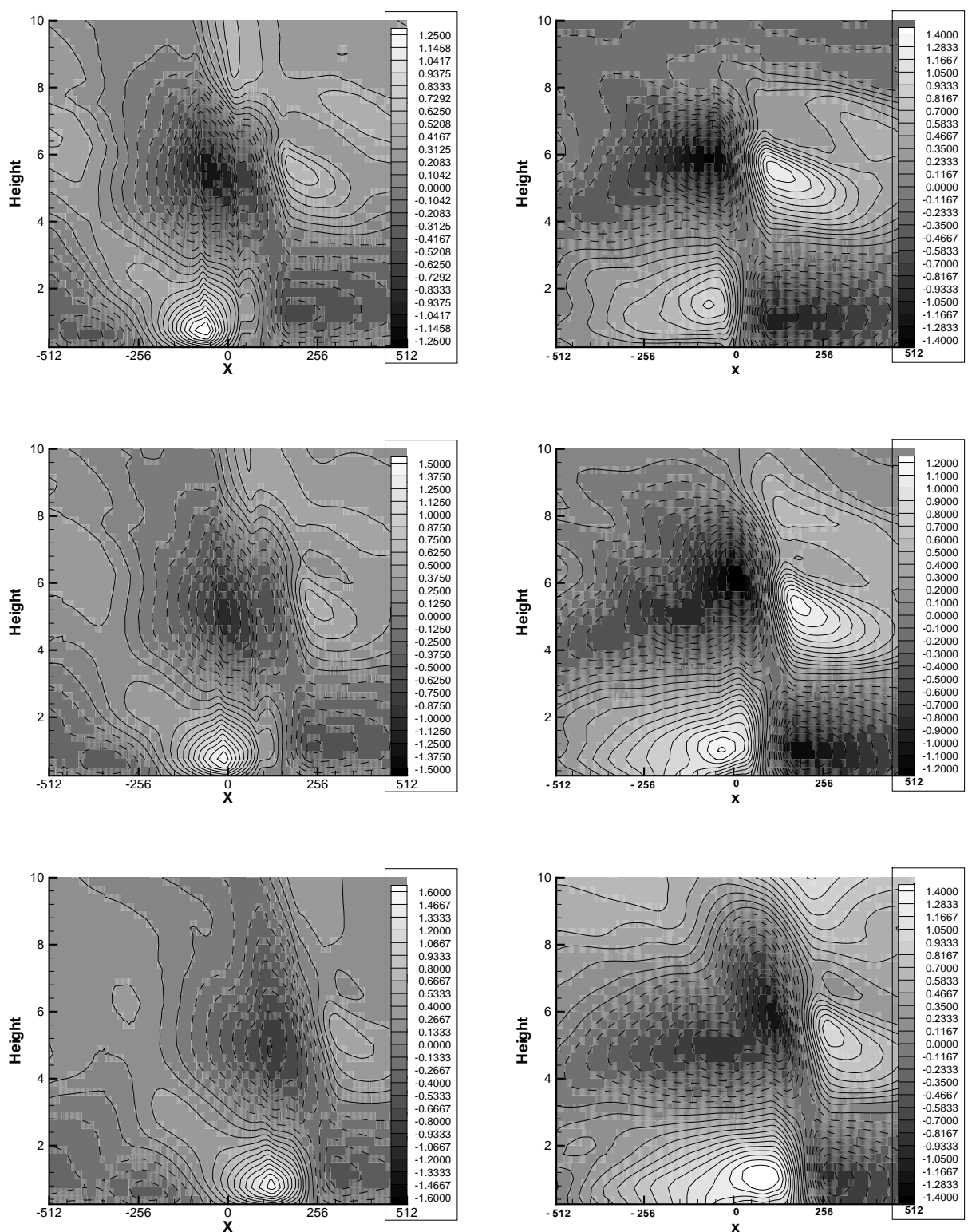

FIG. 4.1. The contours of horizontal velocity $u$. Top: Test with background shear $a=1$; Middle: Test with background shear $a=0.8$; Bottom: Test with background shear $a=0.5$; Left: Velocity from the large scale test; Right: Velocity from the CRM simulation. Note: 1, the units for the x-axis and height are both $\mathrm{km}$ in these plots, rather than the non-dimensional units; 2, a solid contour line represents positive value and a dashed one represents negative value.

in the squall line. Also notice that the strength of these upscale fluxes diminishes with a.

The plots for the eddy flux terms $\overline{\left(\left\langle w^{\prime}\right\rangle\left\langle\theta^{\prime}\right\rangle\right)_{z}}$ and $\overline{\left(\left\langle w^{\prime}\right\rangle\left\langle u^{\prime}\right\rangle\right)_{z}}$ are also shown in figure 4.5. They demonstrate that background shears of the same structure generate 


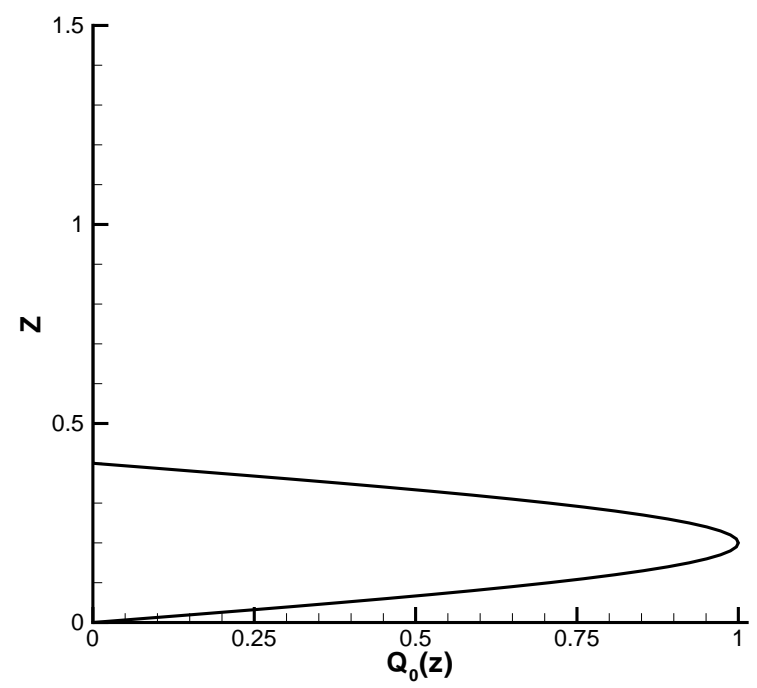

FIG. 4.2. Plot of $Q_{0}(z)$.

the eddy fluxes with the similar structure with amplitude diminishing with $a$. The main forcing from eddies occurs around $2-4 \mathrm{~km}$ high. The eddy momentum flux is associated with acceleration of the shear flow at these levels of $2-4 \mathrm{~km}$; this is the same effect happening qualitatively in the simulations from section 2 at levels of 2-4 $\mathrm{km}$, as the reader can surmise by recalling the calculated eddy momentum flux in the lower left hand panel of Figures 2.8, 2.9, 2.10. This acceleration of the large scale flow is a typical example of the upscale transfer in squall lines of moist available potential energy to the large scale horizontal momentum [27, 39, 40,47]. The strength of the eddy forcing decays as the shear amplitude becomes smaller.

\section{Concluding discussion}

In section 2 we introduced squall lines to the applied mathematics community as multi-scale turbulent traveling waves in the atmosphere and illustrated a surprising new self-similarity principle. New multi-scale asymptotic models that are appropriate for squall lines were developed in section 3 . The new mathematical and physical phenomena in the resulting multi-scale equations were developed in section 4 as well as a simple connection with the squall line simulations in section 2 . There are remarkable important three-dimensional effects in squall lines both in nature and from high resolution numerical simulations involving the effects of mean shear transverse to the propagating turbulent front [19]; there is no existing theory for this behavior. The 3-D version of the new multi-scale models developed in section 3 provides an important starting point for new theories to understand these effects.

As mentioned in the introduction, a major stumbling block in the accurate prediction of weather and short-term climate is the accurate parameterization of moist convection in numerical models $[8,40]$. Recently, an alternative strategy, called superparametrization $[11,12,13,44]$, for including the effects of moist convection in numerical models through explicit turbulent fluxes calculated from a cloud-resolving model has been developed. Superparameterization blends conventional parameteriza- 

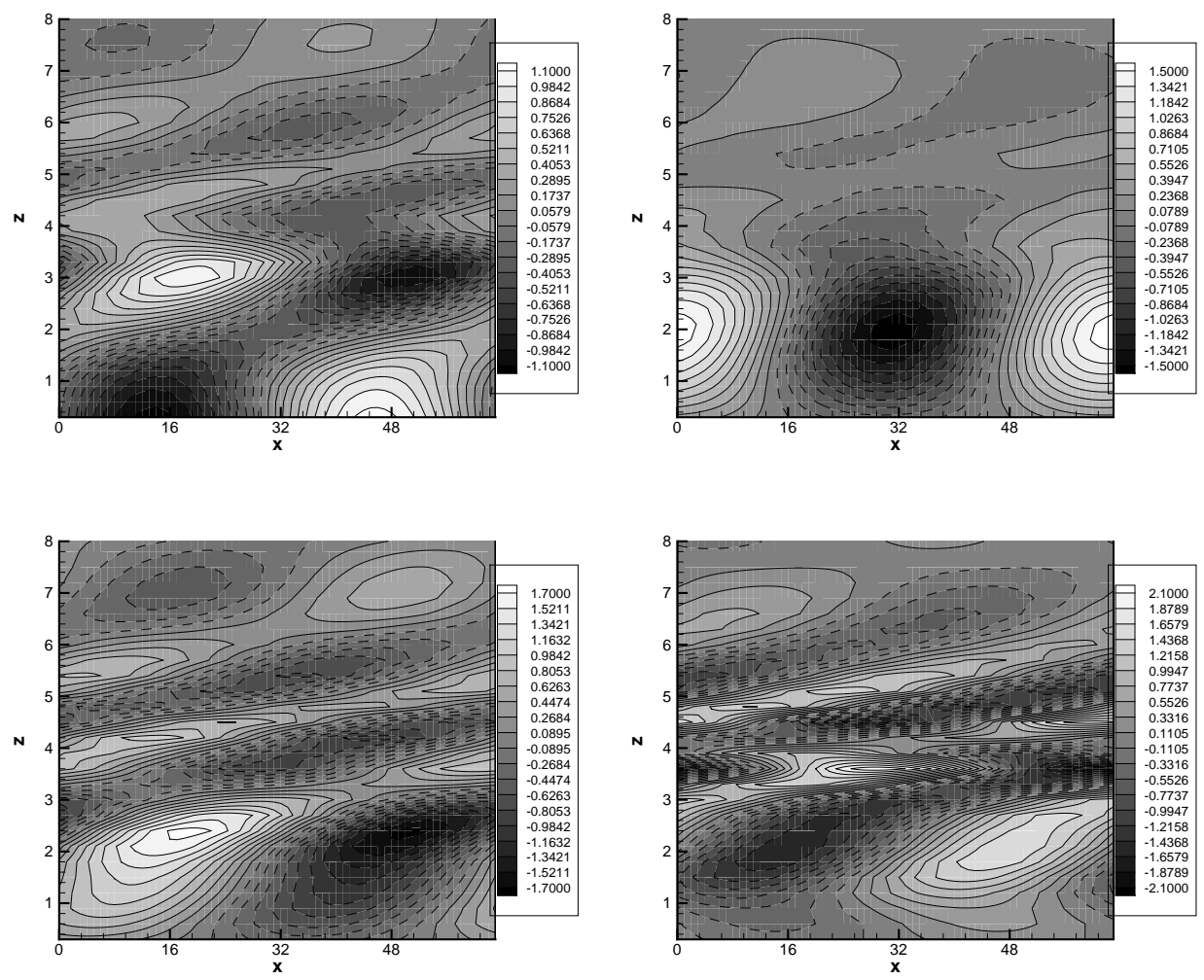

FIG. 4.3. The contours of the variables from the small scale squall-line test. Top left: Horizontal velocity $u^{\prime}$; Top right: Vertical velocity $w^{\prime}$; Bottom left: Potential temperature $\theta^{\prime}$; Bottom right: Vorticity $\omega^{\prime}$. Note: 1, the units for the $x$-axis and height are both $\mathrm{km}$ in these plots, rather that the non-dimensional units; 2, a solid contour line represents positive value and a dashed one represents negative value.

tion on a coarse mesh with detailed cloud-resolving modeling on a finer mesh with an imposed scale gap. This method has yielded promising new results regarding tropical intraseasonal behavior [11, 12, 13, 23], but a systematic formulation and analysis for such superparameterization strategies that might lead to algorithmic improvements are not yet available despite interesting progress in this direction $[13,14,16,22]$. Systematic asymptotic models which make links both with superparameterization and heterogeneous multiscale methods (HMM) [7] but require low Froude numbers have been developed [31]. Recently, the authors with Wojciech Grabowski have developed a new sparse space-time efficient algorithm for superparameterization on mesoscales [48] which exploits small scale self-similarity even though there is a scale gap. The new asymptotic models developed in section 3 of this paper can be applied directly to these algorithms to provide basic understanding of superparameterization as a numerical algorithm. The authors plan to do this in the near future.

Acknowledgement. The research of Andrew Majda is partially supported by ONR N0014-05-1-0164 and NSF DMS-0456713. Yulong Xing has been supported as a postdoctoral fellow through these grants. 

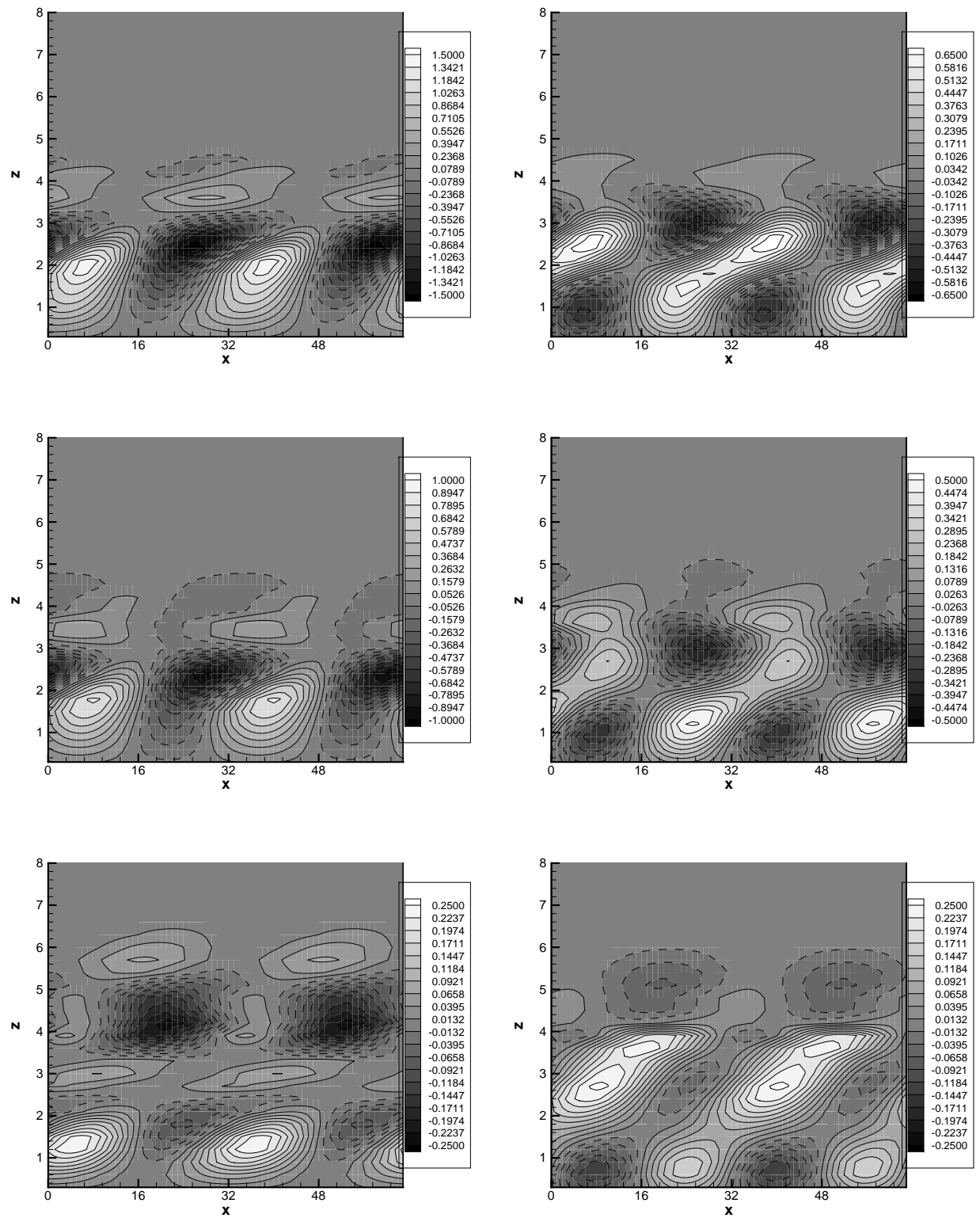

FIG. 4.4. The contours of the variables from the small scale squall-line test. Left: The flux $\overline{\left\langle w^{\prime}\right\rangle\left\langle\theta^{\prime}\right\rangle}$; Right: The flux $\overline{\left\langle w^{\prime}\right\rangle\left\langle u^{\prime}\right\rangle}$; Top: Experiment $a=1$; Middle: Experiment a $=0.8$; Bottom: Experiment $a=0.5$. Note: 1, the units for the $x$-axis and height are both $k m$ in these plots, rather than the non-dimensional units; 2, a solid contour line represents positive value and a dashed one represents negative value.

Appendix A. Non-dimensionalization of the equations. In this appendix, we briefly present the derivation of the non-dimensionalized equations. We start from the governing anelastic equations with the bulk, warm-rain parameterization of moist 

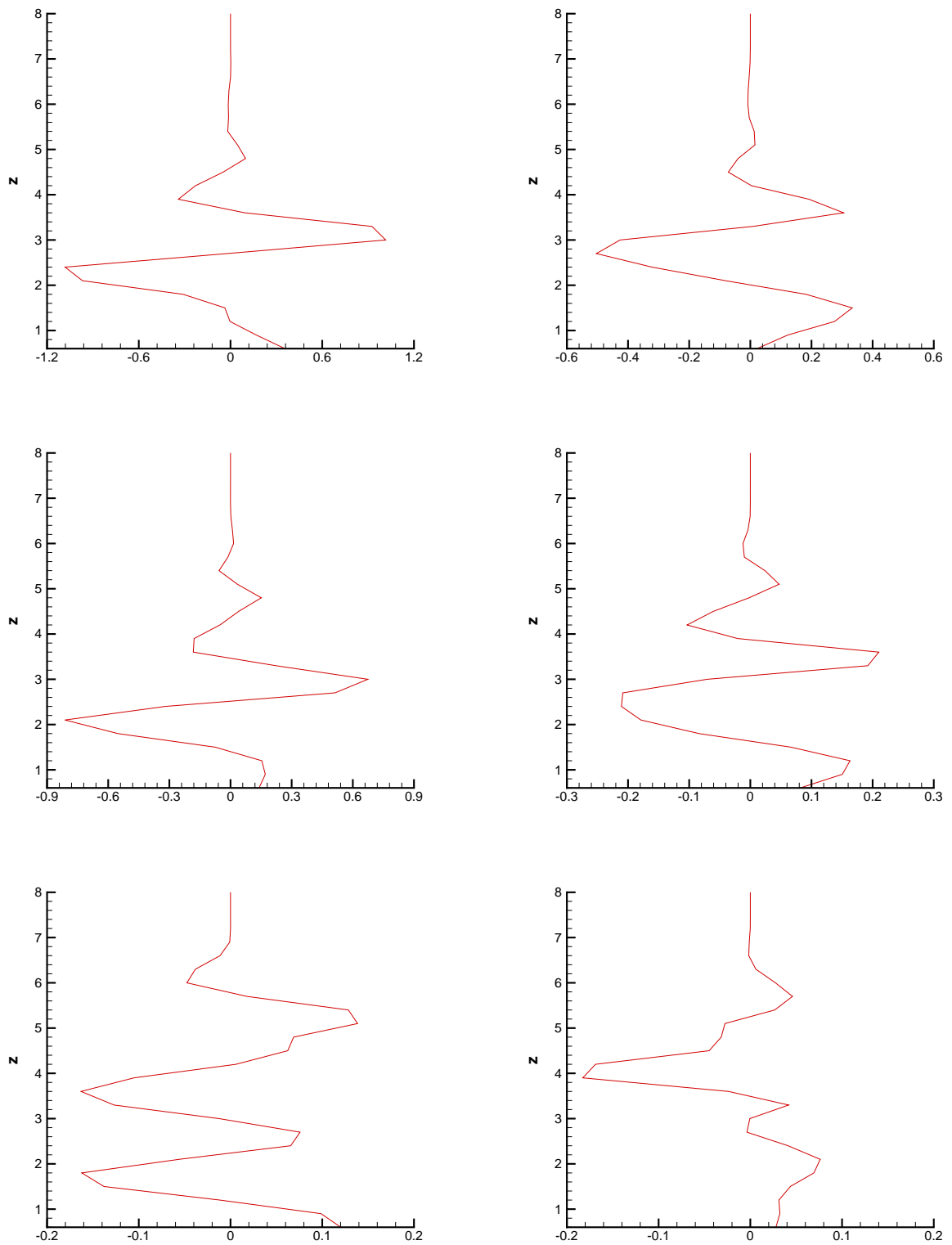

FIG. 4.5. The contours of the eddy flux terms. Left: The flux $\overline{\left(\left\langle w^{\prime}\right\rangle\left\langle\theta^{\prime}\right\rangle\right)_{z}}$; Right: The flux $\overline{\left(\left\langle w^{\prime}\right\rangle\left\langle u^{\prime}\right\rangle\right)_{z}}$; Top: Experiment $a=1$; Middle: Experiment $a=0.8$; Bottom: Experiment $a=0.5$. Note: the units for the $x$-axis and height are both $\mathrm{km}$ in these plots, rather than non-dimensional units.

thermodynamics

$$
\begin{aligned}
\frac{D \mathbf{u}_{h}}{D t} & =-\nabla_{h} p, \\
\frac{D w}{D t} & =-\frac{\partial p}{\partial z}-g \frac{\rho^{\prime}}{\rho_{0}}+\left(\bar{\epsilon} q_{v}-q_{r}-q_{c}\right), \\
\frac{D \theta_{t}}{D t} & =L \frac{\gamma-1}{\gamma} \frac{\theta_{0}}{p_{0}}\left(C_{d}-E_{r}\right), \\
\nabla_{h} \cdot \mathbf{u}_{h}+\rho^{-1}(\rho w)_{z} & =0
\end{aligned}
$$


and the cloud dynamic equations are given by

$$
\begin{aligned}
\frac{D q_{v}}{D t} & =-C_{d}+E_{r}, \\
\frac{D q_{c}}{D t} & =C_{d}-A_{r}, \\
\frac{D q_{r}}{D t}-\frac{1}{\rho} \frac{\partial}{\partial z}\left(\rho V_{t} q_{r}\right) & =A_{r}-E_{r},
\end{aligned}
$$

with

$$
\frac{D}{D t}=\frac{\partial}{\partial t}+\mathbf{u}_{h} \cdot \nabla_{h}+w \frac{\partial}{\partial z}
$$

Here $\mathbf{u}_{h}=(u, v)$ is the horizontal velocity, $w$ is the vertical velocity, $p$ is pressure, $g$ is the acceleration of gravity, $\theta$ is the potential temperature, $q_{v}, q_{c}, q_{r}$ are water vapor, cloud water, and rain water mixing ratios, respectively, and $\gamma$ is the isentropic exponent. $\bar{\epsilon}+1$ is the ratio of gas constants of water vapor and dry air, $L$ denotes the latent heat of condensation, $p_{0}=10^{5} \mathrm{~kg} \mathrm{~m}^{-2}$, and $\rho_{0}=1 \mathrm{~kg} \mathrm{~m}^{-3}$. We assume

$$
\begin{aligned}
\theta_{t} & =\theta_{0}+\theta_{b g}(z)+\theta, \\
\theta_{0} & =300 \mathrm{~K}, \\
\frac{d \theta_{b g}}{d z} & =\frac{N^{2}(z) \theta_{0}}{g}, \\
\frac{\rho^{\prime}}{\rho_{0}} & =-\frac{\theta}{\theta_{0}}
\end{aligned}
$$

where

$$
N(z)=-\frac{g}{\rho} \frac{d \rho}{d z}
$$

is the Brunt-Väisälä buoyancy frequency. Thus the system (A.1) becomes

$$
\begin{aligned}
\frac{D \mathbf{u}_{h}}{D t} & =-\nabla_{h} p, \\
\frac{D w}{D t} & =-\frac{\partial p}{\partial z}+g \frac{\theta}{\theta_{0}}+\left(\bar{\epsilon} q_{v}-q_{r}-q_{c}\right), \\
\frac{D \theta}{D t}+w \frac{d \theta_{b g}}{d z} & =L \frac{\gamma-1}{\gamma} \frac{\theta_{0}}{p_{0}}\left(C_{d}-E_{r}\right), \\
\nabla_{h} \cdot \mathbf{u}_{h}+\rho^{-1}(\rho w)_{z} & =0 .
\end{aligned}
$$

We introduce the following non-dimensional units

$$
\begin{aligned}
\mathbf{u} & =U \widehat{\mathbf{u}}, \quad w=W \widehat{w}, \quad \theta=\Theta \widehat{\theta}, \\
\mathbf{x} & =H \widehat{\mathbf{x}}, \quad z=H \widehat{z}, \quad t=T \widehat{t}, \\
p & =P \widehat{p}, \quad N(z)=N_{0} \widehat{N}(z), \\
q_{v} & =q_{v s}^{*} \widehat{q_{v}}, \quad q_{c}=q_{v s}^{*} \widehat{q_{c}}, \quad q_{r}=q_{v s}^{*} \widehat{q_{r}}, \\
C_{d} & =\frac{q_{v s}^{*}}{T} \widehat{C_{d}}, \quad E_{r}=\frac{q_{v s}^{*}}{T} \widehat{E_{r}}, \quad A_{r}=\frac{q_{v s}^{*}}{T} \widehat{A_{r}},
\end{aligned}
$$


with

$$
\begin{aligned}
U & =W=\frac{H}{T}, \quad \Theta=\frac{N_{0} \theta_{0} H}{g T}, \\
q_{v s}^{*} & =\left(\frac{\rho_{v, s a t}}{\rho_{d}}\right)_{r e f}, \quad P=\frac{W H}{T}=\frac{H^{2}}{T^{2}} .
\end{aligned}
$$

Substituting these non-dimensional units in (A.4), we obtain

$$
\begin{aligned}
& \frac{\widehat{D} \widehat{\mathbf{u}}}{D \widehat{t}}=-\widehat{\nabla}_{h} \widehat{p}, \\
& \frac{\widehat{D} \widehat{w}}{D \widehat{t}}=-\frac{\partial \widehat{p}}{\partial \widehat{z}}+N_{0} T \widehat{\theta}+\frac{T^{2}}{H} q_{v s}^{*}\left(\bar{\epsilon} \widehat{q_{v}}-\widehat{q_{r}}-\widehat{q_{c}}\right), \\
& \frac{\widehat{D} \widehat{\theta}}{D \widehat{t}}+\frac{H}{\Theta} \frac{N^{2} \theta_{0}}{g} \widehat{w}=\frac{q_{v s}^{*}}{\Theta} L \frac{\gamma-1}{\gamma} \frac{\Theta \widehat{\theta_{0}}}{P \widehat{p_{0}}}\left(\widehat{C_{d}}-\widehat{E_{r}}\right) \text {, } \\
& \widehat{\nabla}_{h} \cdot \widehat{\mathbf{u}}+\rho^{-1}(\rho \widehat{w})_{\widehat{z}}=0, \\
& \frac{D \widehat{q_{v}}}{D \widehat{t}}=-\widehat{C_{d}}+\widehat{E_{r}}, \\
& \frac{D \widehat{q_{c}}}{D \widehat{t}}=\widehat{C_{d}}-\widehat{A_{r}}, \\
& \frac{D \widehat{q_{r}}}{D \widehat{t}}-\frac{1}{\rho} \frac{\partial}{\partial \widehat{z}}\left(\rho \widehat{V_{t}} \widehat{q_{r}}\right)=\widehat{A_{r}}-\widehat{E_{r}} .
\end{aligned}
$$

Note that the coefficient of $\widehat{w}$ in (A.6c) becomes

$$
\frac{H}{\Theta} \frac{N^{2} \theta_{0}}{g}=\frac{N_{0} T}{\Theta} \frac{N_{0} \theta_{0} H}{g T} \hat{N}^{2}=\frac{N_{0} T}{\Theta} \Theta \hat{N}^{2}=N_{0} T \hat{N}^{2}(z) .
$$

We now fix the reference magnitudes as following

$$
\begin{aligned}
H & =10 \mathrm{~km}=10^{4} \mathrm{~m}, \\
T & =15 \mathrm{~min}=900 \mathrm{~s}, \\
N_{0} & =10^{-2} \mathrm{~s}^{-1}, \\
\Theta & =\frac{N \theta_{0} H}{g T}=\frac{0.01 \times 300 \times 10^{4}}{10 \times 900} \simeq 3 \mathrm{~K}, \\
P & =\frac{W H}{T}=\frac{H^{2}}{T^{2}}=100, \\
q_{v s}^{*} & =0.01, \\
\gamma & =1.4 .
\end{aligned}
$$

Thus, we can compute the coefficients in (A.6)

$$
\begin{aligned}
N_{0} T & =0.01 \times 900 \simeq 10=\epsilon^{-1}, \\
\frac{T^{2}}{H} q_{v s}^{*} & =\frac{900^{2}}{10^{4}} \times 0.01 \simeq 1, \\
\frac{H}{\Theta} \frac{N^{2} \theta_{0}}{g} & =N_{0} T \hat{N}^{2}(z) \simeq \epsilon^{-1} \hat{N}^{2}(z), \\
\frac{q_{v s}^{*}}{\Theta} L \frac{\gamma-1}{\gamma} \frac{\Theta}{P \widehat{\theta_{0}}}\left(\widehat{C_{d}}-\widehat{E_{r}}\right) & \simeq \epsilon^{-1} \hat{L} \frac{\widehat{\theta_{0}}}{\widehat{p_{0}}}\left(\widehat{C_{d}}-\widehat{E_{r}}\right),
\end{aligned}
$$


where $\hat{L}$ is order 1 latent heat prefactor, and $\epsilon=0.1$. Therefore, the system (A.6) becomes (the hat is dropped for convenience)

$$
\begin{aligned}
\frac{D \mathbf{u}_{h}}{D t} & =-\nabla_{h} p \\
\frac{D w}{D t} & =-p_{z}+\epsilon^{-1} \theta+\left(\bar{\epsilon} q_{v}-q_{r}-q_{c}\right) \\
\frac{D \theta}{D t}+N^{2}(z) \epsilon^{-1} w & =\epsilon^{-1} L \frac{\theta_{0}}{p_{0}}\left(C_{d}-E_{r}\right) \\
d i v_{h} \mathbf{u}_{h}+\rho^{-1}(\rho w)_{z} & =0 \\
\frac{D q_{v}}{D t} & =-C_{d}+E_{r} \\
\frac{D q_{c}}{D t} & =C_{d}-A_{r} \\
\frac{D q_{r}}{D t}-\frac{1}{\rho} \frac{\partial}{\partial z}\left(\rho V_{t} q_{r}\right) & =A_{r}-E_{r} .
\end{aligned}
$$

\section{REFERENCES}

[1] A. Bayliss, M. Gunzburger and E. Turkel, Boundary conditions for the numerical solution of elliptic equations in exterior regions, SIAM J. Appl. Math., 42, 430-451, 1982.

[2] J.A. Biello and A.J. Majda, A new multiscale model for the Madden-Julian oscillation., J. Atmos. Sci., 62, 1694-1721, 2005.

[3] J.A. Biello and A.J. Majda, Modulating synoptic scale convective activity and boundary layer dissipation in the IPESD models of the Madden-Julian oscillation, Dyn. Atmos. Oceans, $42,152-215,2006$.

[4] J.A. Biello and A.J. Majda, Intraseasonal multi-scale moist dynamics of the tropical troposphere, Commun. Math. Sci., in press, 2009.

[5] J.A. Biello, A.J. Majda and M.W. Moncrieff, Meridional momentum flux and superrotation in the multi-scale IPESD MJO model, J. Atmos. Sci., 64, 1636-1651, 2007.

[6] N.A. Crook and M.W. Moncrieff, The effect of large-scale convergence on the generation and maintenance of deep moist convection, J. Atmos. Sci., 45, 3606-3624, 1988.

[7] W. E and B. Engquist, The heterogeneous multiscale methods, Commun. Math. Sci., 1, 87-132, 2003.

[8] K.A. Emanuel, Atmospheric Convection, Oxford University Press, 1994.

[9] R.G. Fovell and P.H. Tan, The temporal behavior of numerically simulated multicell-type storms. part II: the convective cell life cycle and cell regeneration, Mon. Wea. Rev., 126, 551-577, 1998.

[10] R.G. Fovell and Y. Ogura, Numerical simulation of a midlatitude squall line in two dimensions, J. Atmos. Sci., 45, 3846-3879, 1988.

[11] W.W. Grabowski, Coupling cloud processes with the large-scale dynamics using the cloudresolving convection parameterization (CRCP), J. Atmos. Sci., 58, 978-997, 2001.

[12] W.W. Grabowski, MJO-like coherent structures: sensitivity simulations using the cloudresolving convection parameterization (CRCP), J. Atmos. Sci., 60, 847-864, 2003.

[13] W.W. Grabowski, An improved framework for superparameterization, J. Atmos. Sci., 61, 19401952, 2004.

[14] W.W. Grabowski, Comments on "preliminary tests of multiscale modeling with a twodimensional framework: sensitivity to coupling methods", Mon. Wea. Rev., 134, 2021-2026, 2006.

[15] W.W. Grabowski and M.W. Moncrieff, Large-scale organization of tropical convection in twodimensional explicit numerical simulations, Q. J. Roy. Met. Soc., 127, 445-468, 2001.

[16] W.W. Grabowski and M.W. Moncrieff, Moisture-convection feedback in the tropics, Q. J. Roy. Met. Soc., 130, 3081-3104, 2004.

[17] W.W. Grabowski and P.K. Smolarkiewicz, Two-time-level semi-Lagrangian modeling of precipitating clouds, Mon. Wea. Rev., 124, 487-497, 1996.

[18] P. Hazel, Numerical studies of the stability of inviscid parallel shear flows, J. Fluid Mech., 51, 39C62, 1972. 
[19] R.A. Houze, Mesoscale convective systems, Rev. Geophys., 42, RG4003, doi:10.1029/2004RG 000150, 2004

[20] H.H. Hendon and M.L. Salby, The life cycle of the Madden-Julian oscillation, J. Atmos. Sci., 51, 225-2237, 1994.

[21] R.H. Johnson and P.J. Hamilton, The relationship of surface pressure features to the precipitation and airflow structure of an intense midlatitude squall line, Mon. Wea. Rev., 116, $1444-1473,1988$.

[22] J.H. Jung and A. Arakawa, Preliminary tests of multiscale modeling with a twodimensional framework: sensitivity to coupling methods, Mon. Wea. Rev., 133, 649-662, 2005.

[23] M. Khairoutdinov, D. Randall and C. DeMott, Simulations of the atmospheric general circulation using a cloud-resolving model as a superparameterization of physical processes, J. Atmos. Sci., 62, 2136-2154, 2005.

[24] R. Klein, Asymptotic analyses for atmospheric flows and the construction of asymptotically adaptive numerical methods, Z. Angew. Math. Mech., 80, 765-777, 2000.

[25] R. Klein, An applied mathematical view of meteorological modeling, Applied Mathematics Entering the 21st Century: Invited Talks from the ICIAM 2003 Congress, J. M. Hill and R. Moore, Eds., Proceedings in Appl. Math., SIAM, 116, 227-289, 2004.

[26] R. Klein and A.J. Majda, Systematic multiscale models for deep convection on mesoscales, Theo. Comp. Fluid Dyn., 20, 525-551, 2006.

[27] J.P. Lafore and M.W. Moncrieff, A numerical investigation of the organization and interaction of the convective and stratiform regions of tropical squall lines, 46, 521-544, 1989.

[28] Y.L. Lin, Two-dimensional response of a stably stratified shear flow to diabatic heating, J. Atmos. Sci., 44, 1375-1393, 1987.

[29] F.B. Lipps and R.S. Hemler, A scale analysis of deep moist convection and some related numerical calculations, J. Atmos. Sci., 39, 2192-2210, 1982.

[30] A.J. Majda, Introduction to PDEs and Waves for the Atmosphere and Ocean, Courant Lecture Notes Math. AMS/CIMS, New York, 9, 2003.

[31] A.J. Majda, Multiscale models with moisture and systematic strategies for superparameterization, J. Atmos. Sci., 64, 2726-2734, 2007.

[32] A.J. Majda, New multiscale models and self-similarity in tropical convection, J. Atmos. Sci., 64, 1393-1404, 2007.

[33] A.J. Majda and J.A. Biello, A multiscale model for tropical intraseasonal oscillation, Proc. Natl. Acad. Sci. USA, 101, 4736-4741, 2004.

[34] A.J. Majda and R. Klein, Systematic multiscale models for the tropics, J. Atmos. Sci., 60, 393-408, 2003.

[35] A.J. Majda, M. Mohammadian and Y. Xing, Vertically sheared horizontal flow with mass sources: a canonical balanced model, Geophys. Astrophys. Fluid Dyn., 202, 543-591, 2008.

[36] A.J. Majda, M. Mohammadian and Y. Xing, Moist multiscale equations for the hurricane embryo, J. Fluid Mech., submitted, 2009.

[37] A.J. Majda and S. Stechmann, A simple dynamic model with features of convective momentum transport, J. Atmos. Sci., 66, 373-392, 2009.

[38] B.E. Mapes, S. Tulich, J.L. Lin and P. Zuidema, The mesoscales convection life cycle: building block or prototype for large-scale tropical waves? Dyn. Atmos. Oceans, 42, 3-29, 2006.

[39] M.W. Moncrieff, Organized convective systems: archetypal dynamical models, mass and momentum flux theory, and parameterization, Q. J. R. Meteorol. Soc., 118, 819-850, 1992.

[40] M.W. Moncrieff and C. Liu, Representing convective organization in prediction models by a hybrid strategy, J. Atmos. Sci., 63, 3404-3420, 2006.

[41] M.W. Moncrieff, M. Shapiro, J. Slingo and F. Molteni, Collaborative research at the intersection of weather and climate, WMO Bulletin, 56, 206-211, 2007.

[42] T. Nakazawa, Tropical super clusters within intraseasonal variations over the western Pacific, J. Meteor. Soc. Japan, 66, 823-839, 1988.

[43] R.E. Pandya and D.R. Durran, The influence of convectively generated thermal forcing on the mesoscale circulation around squall lines, J. Atmos. Sci., 53, 2924-2951, 1996.

[44] D. Randall, M. Khairoutdinov, A. Arakawa and W. Grabowski, Breaking the cloud parameterization deadlock, Bull. Amer. Meteor. Soc., 84, 1547-1564, 2003.

[45] R. Rotunno, J.B. Klemp and M.L. Weisman, A theory for strong, long-lived squall lines, J. Atmos. Sci., 45, 463-485, 1988.

[46] M. Wheeler and G.N. Kiladis, Convectively coupled equatorial waves: analysis of clouds and temperature in the wave-number-frequency domain, J. Atmos. Sci., 56, 374-399, 1999. 
[47] X. Wu and M.W. Moncrieff, Collective effects of organized convection and their approximation in general circulation models, J. Atmos. Sci., 53, 1477-1495, 1996.

[48] Y. Xing, A.J. Majda and W.W. Grabowski, New efficient sparse space-time algorithms for superparameterization on mesoscales, Mon. Wea. Rev., in press, 2009.

[49] M.J. Yang and R.A. Houze, Multicell squall-line structure as a manifestation of vertically trapped gravity waves, Mon. Wea. Rev., 123, 641-661, 1995. 